\title{
Geospatial modelling on the spread and dynamics of 154 day outbreak of the novel coronavirus (COVID-19) pandemic in Bangladesh towards vulnerability zoning and management approaches
}

\author{
Md. Rejaur Rahman ${ }^{1}$ (D) A. H. M. Hedayutul Islam ${ }^{1}$ - Md. Nazrul Islam²
}

Received: 24 August 2020 / Accepted: 1 September 2020 / Published online: 9 September 2020

(c) Springer Nature Switzerland AG 2020

\begin{abstract}
The novel COVID-19 is a worldwide transmitted pandemic and has received global attention. Since there is no effective medication yet, to minimize and control the transmission of the COVID-19, non-pharmaceutical interventions (NPIs) are followed globally. However, for the implementation of needful NPIs through effective management strategies and planning, space-time-based information on the nature, magnitude, pattern of transmission, hotspots, the potential risk factors, vulnerability, and risk level of the pandemic are important. Hence, this study was an attempt to in-depth assess and analyze the COVID-19 outbreak and transmission dynamics through space and time in Bangladesh using 154 day real-time epidemiological data series. District-level data were analyzed for the geospatial analysis and modelling using GIS. Getis-Ord $\mathrm{Gi}^{*}$ statistics was applied for the hotspot analysis, and on the other hand, the analytical hierarchy process-based weighted sum method (AHP-WSM) was used for the modelling of vulnerability zoning of COVID-19. In Bangladesh, the status of the pandemic COVID-19 still is in exposure level. Disease transmitted at a high rate (20.37\%), and doubling time of the cases were 11 days (latest week of the study period). The fatality rate was comparatively low (1.3\%), and the recovery rate was about $57.50 \%$. Geospatial analysis exhibits the disease propagates from the central parts, and Dhaka was the most exposed district followed by Chattogram, Narayanganj, Cumilla, and Bogra. A single strong clustering pattern in the central part, which spread out mainly to the south-eastern part, was identified as a prime hotspot in both the cases and deaths distributions. Additionally, potential linkages between the transmission of disease and the selected factors that gear up the spreading of the disease were identified. The central, eastern, and south-eastern parts were recognized as high vulnerable zone, and conversely, the western, south-western, north-western, and north-eastern parts as medium vulnerable zone. The vulnerable zoning exercise made it possible to identify vulnerable areas with the different magnitude that require urgent intervention through proper management and action plan, and accordingly, comprehensive management strategies were anticipated. Thus, this study will be a useful guide towards understanding the space-time-based investigations and vulnerable area delineation of the COVID-19 and assist to formulate an effective management action plan to reduce and control the disease propagation and impacts. By appropriate adjustment of some factors with local relevance, COVID-19 vulnerability zoning derived here can be applied to other regions, and generally can be used for any other infectious disease. This method was applied at a regional scale, but the availability of larger scale data of the determining factors could be applied in small areas too, and accordingly, management strategies can be formulated.
\end{abstract}

Keywords COVID-19 · Geospatial modelling · GIS and hotspots · Vulnerability zoning · Management strategies · Bangladesh

\section{Introduction}

The present world is facing a unique health problem due to an outbreak of a novel coronavirus disease known as

Md. Rejaur Rahman

rejaur2001@yahoo.com

Extended author information available on the last page of the article COVID-19. This is a great threat to humankind. This virus has extended almost all of the populated world and the impact of the pandemic is being felt in almost every aspect 
of our lives. COVID-19 is a respiratory infectious disease, first recognized in the city Wuhan, China on 31 December 2019 and the first reported death from COVID-19 also in China in January 2020 (WHO 2020a). It was also reported that on 13 January 2020, the first case outside of China was identified in Thailand (Hui 2020). It is caused by a new virus from the SARS (Severe Acute Respiratory Syndrome) family called severe acute respiratory syndrome coronavirus (SARS-CoV-2) or coronavirus 2019-nCoV or COVID-19 (WHO 2020b). It is transmitted from person-to-person via airborne respiratory droplets, direct contact with body fluids or secretions, or through contaminated objects (Xu et al. 2020). Viewing the spread of COVID-19 outside of China, on 30 January 2020 World Health Organization (WHO), has declared the outbreak as a Public Health Emergency of International Concern (PHEIC) (WHO 2020c). WHO has suggested further to follow the practices to interrupt humanto-human spreading and preventing the level of spreading, and also avoiding further international spread on 01 March 2020 (Böhmer et al. 2020). Later, on 11 March 2020, WHO announced COVID-19 outbreak as a world pandemic when it spreads to 114 countries with 118,000 infections and 4291 deaths (WHO 2020d). As of 29 August 2020, there were 25,016,706 confirmed cases and 843,120 deaths recorded worldwide due to COVID-19 (WHO 2020e). On that day, the USA, Brazil, India, Russia, Peru, South Africa, Colombia, Mexico, Spain, and Chile were in the top ten worst listed countries based on confirmed cases. On the contrary, according to the death toll, the top ten listed countries were USA, Brazil, India, Mexico, UK, Italy, France, Spain, Peru, and Iran (WHO 2020e). Again, estimation through model projection depicted that if there is no implementation of interventions against COVID-19 spreading, about 40 million deaths may occur globally this year (Walker et al. 2020). Moreover, statistics of COVID-19 cases depict that some countries are experiencing the second wave of attacking of the virus, e.g., a new outbreak of COVID-19 cases had infected scores of people in China's capital, Beijing on 19 June 2020 (CGTN 2020), South Korea confirms the second wave of infections on 22 June 2020, the USA preparing for the second wave of coronavirus as 12 States hit daily records on 22 June 2020 , and health experts in Britain were warning ministers to prepare for the next wave of virus attack on 24 June 2020 (BBC 2020). A century ago, the second wave of infection of Spanish flu was more severe than the first (Kenner 2010). Besides, according to the reports of United Nations Development Programme (UNDP), as of mid-May 2020, the number of daily deaths due to COVID-19 was higher than the number of deaths due to common causes such as malaria, suicide, road traffic accidents, and HIV/AIDS (UNDP 2020). Thus, COVID-19 pandemic may force to face a big challenge to comprehensive healthcare systems and managements, society, economy, environment, and international relations in the coming days. Additionally, the COVID-19 will also effect on the achievement of the United Nations (UN) sustainable development goals (SDGs) for health, economic, and social development towards sustainable development (UNDP 2015; Zhou et al. 2020). Moreover, the extensive spread of COVID-19 and its consequences all over the globe has led societies to suffer severe health-related problems, including fear, panic, anxiety, depression, racial discrimination, and intolerance (Bodrud-Doza 2020; Khan et al. 2020a).

Like other countries, Bangladesh is not an exception, and on 13 June 2020, it exceeds China considering the total number of confirmed cases (recorded a total number of 84,379 cases, while China experienced 83,075), and as of 29 August 2020, country's rank was 15th in the list (with 308,925 confirmed cases) of COVID-19 worst-hit countries (WHO 2020e). Up to now, for the prevention and treatment of COVID-19 disease, there is no effective medication available and therefore, to control the transmissions of COVID19 virus, mainly community-based and non-pharmaceutical intervention (NPI), including isolation, quarantine, social distancing, protection of vulnerable groups, school closures, restricting public events, and lockdown are followed globally. These measures are mainly to minimize personto-person spreads of COVID-19 through social distancing and protection (Ainslie et al. 2020; Ferguson et al. 2020; Chowdhury et al. 2020). Viewing the severity of COVID19 till now, it is necessary to work to control the spreading of COVID-19 within a least possible time and to minimize the impact of the disease on the inhabitants. Based on the past experiences of other epidemics, information from published literature, and measures taken by different countries related to COVID-19, two possible ways or approaches can be implemented to control the disease (Chinazzi et al. 2020; Zhu et al. 2020a; Aleta and Moreno 2020; Li et al. 2020; Xiong et al. 2020). Development of new vaccines and medication, i.e., pharmaceutical interventions (PI) approach could be the one effective way to stop the infection, but generally needs much of time to introduce new vaccines for the new virus. Since COVID-19 virus is transmitted from person-to-person, the nature of the spreading, spatial pattern of spreading, identification of vulnerable people, and vulnerability through spatial analysis and modelling, which can help the governments, policymakers, and other decision-making personnel to formulate effective planning and control measures for the proper lockdown, isolation, protection of vulnerable groups, and restricting public events, i.e., non-pharmaceutical intervention (NPI) approach could be another way to minimize and control the rate of infection or delay the disease spreading. To control COVID-19 emergencies through NPI, Geographic Information System (GIS) along with geospatial data can be played an important role, since GIS is competent and a widespread technological way for capturing, preparing, managing, aggregating, 
analyzing, and visualizing the multi-source, non-geospatial, and geospatial data (Rahman and Saha 2008; Rahman et al. 2014, 2015; Rahman and Lateh 2016a, b; Rahman and Lateh 2017). Using GIS, it is also possible to do spatial tracking, identification of the geographical pattern of transmission, spatial segmentation for vulnerability and risk assessment, spatial modelling, and visualization of any event that can provide compact spatial information support for decisionmaking, measures formulation, and effectiveness assessment of the event to prevent and control (Zhou et al. 2020;). It is long back that in 1854, mapping and spatial analysis was used by the British physician John Snow to detect and analyze a cholera outbreak in London. The findings of that study helped to save many lives and assisted to formulate a modern water and waste management systems in London. Later, it was followed by other cities and made a significant change in general public health around the world (Brody 2003).

The analysis of the pandemic situation over the space and time is crucial to understand the nature, magnitude, geographical pattern of transmission, the potential risk factors, vulnerability and risk level, and hotspots of infection (Cliff and Ord 1981; Kandwal et al. 2009; Lyseen et al. 2014). Spatiotemporal analysis of the COVID-19 outbreak is also important to formulate a comprehensive policy to control the adverse situation of the pandemic using the resources available. Although spatial and spatio-temporal autocorrelation and analysis are important in epidemiology (Cliff and Ord 1981) and, past 2 decades, spatial epidemiology has been involved in and has found a great response in medical applied research (Kirby et al. 2017); however, after the outbreak of COVID-19, the geospatial technologies, such as GIS, is treated only marginally. Literature supports that some certain studies were carried out over different parts of the world regarding the analysis of COVID-19 spatially (Zhou et al. 2020; Fan et al. 2020, Gatto et al. 2020, Murugesan et al. 2020; Kamel-Boulos and Geraghty 2020; Meyer et al. 2017; Mo et al. 2020; Roy et al. 2020). Particularly, a comprehensive study on the nature of spatial spreading, geospatial pattern of transmission, vulnerability, and risk level along with hot spots analysis of COVID-19 were not widely analyzed (Ramírez-Aldana et al. 2020; Huang et al. 2020; Jia et al 2020; Kang et al. 2020; Melin et al. 2020; Ghosh et al. 2020; Mishra et al. 2020; Acharya and Porwal 2020). Conversely, in case of Bangladesh, in-depth study of COVID-19 using geospatial approach was very rare (Sakamoto et al. 2020; Masrur et al. 2020) and which were available mostly epidemiological, temporal, and modelling based and some of those mainly highlighted the impacts of COVID-19 on the economy, society, health, and environment (Anwar et al. 2020; Bodrud-Doza 2020; Hossain et al. 2020; Jahan et al. 2020; Khan et al. 2020a, b; Khan et al. 2020a; Zabir et al. 2020). Considering the above facts, the objectives of this study were fixed to (i) find out the spreading nature of
COVID-19 in 154 days of the outbreak of the disease in Bangladesh concerning the interventions which have been taken and implemented, (ii) find out the spatio-temporal patterns of the disease for exploring the spatial epidemic dynamics, (iii) identify the hotspots of the COVID-19 at the district level, (iv) correlate the spatial pattern of the disease with some bio-socio-economic factors to generate a COVID19 vulnerability zoning (Cov19VZ) map using GIS-aided method, and, finally, (v) formulate a management strategy based on the vulnerability zoning that can be used as a basis for the next-step planning and prevention the spread of the infectious diseases.

\section{Data used and methods}

\section{Study area}

In this study, we try to analyze the spatio-temporal pattern of COVID-19 propagation in Bangladesh using available daily and district-level data. Thus, Bangladesh was selected as a study area and the districts, which are the second level of the administrative unit from the top were selected as the spatial units for this study. The geographical boundary of Bangladesh is between $20^{\circ} 34^{\prime} \mathrm{N}-26^{\circ} 38^{\prime} \mathrm{N}$ latitude and $88^{\circ} 01^{\prime} \mathrm{E}-92^{\circ} 41^{\prime} \mathrm{E}$ longitude (Fig. 1), and occupied an area of total 144,000 km² (Rahman and Lateh 2016a). Bangladesh is situated in the Indian subcontinent and surrounded by India in the north, east, and west and by Myanmar in the southeast. The southern part of the country is surrounded by the Bay of Bengal. There are 64 districts in Bangladesh under 8 divisions (first level of the administrative unit from the top). The divisions are Dhaka, Chattogram, Khulna, Rajshahi, Rangpur, Barishal, Mymensingh, and Sylhet with consist of 13, 11, 10, 8, 8, 6, 4, and 4 districts, respectively (Fig. 1). Climatically, the country belongs to subtropical region and has a typical monsoon climate that characterized by wide seasonal variations in rainfall, moderately warm temperature, and high humidity. The total population of the country is about 160 million, and the population density is about 1125 people per square kilometres (BBS 2015c). Dhaka, the capital, is one of the highest densely populated areas of the world $(23,234$ people per square kilometres, BBS 2015c). The urban population in the country is about $21 \%$ of the total population, and about $28 \%$ of the urban population live in Dhaka alone. Dhaka, Chattogram, Narayanganj, Gazipur, and Khulna are the main business hub of the country. The economy of the country is largely dependent on foreign remittances and export earnings from the garments industry. About 41 and 37\% of the total migrant household (international migrant family from Bangladesh) are in Dhaka and Chattogram divisions, respectively (BBS 2015e). Again, 49 and $11 \%$ industry/factory workers are engaged in Dhaka 


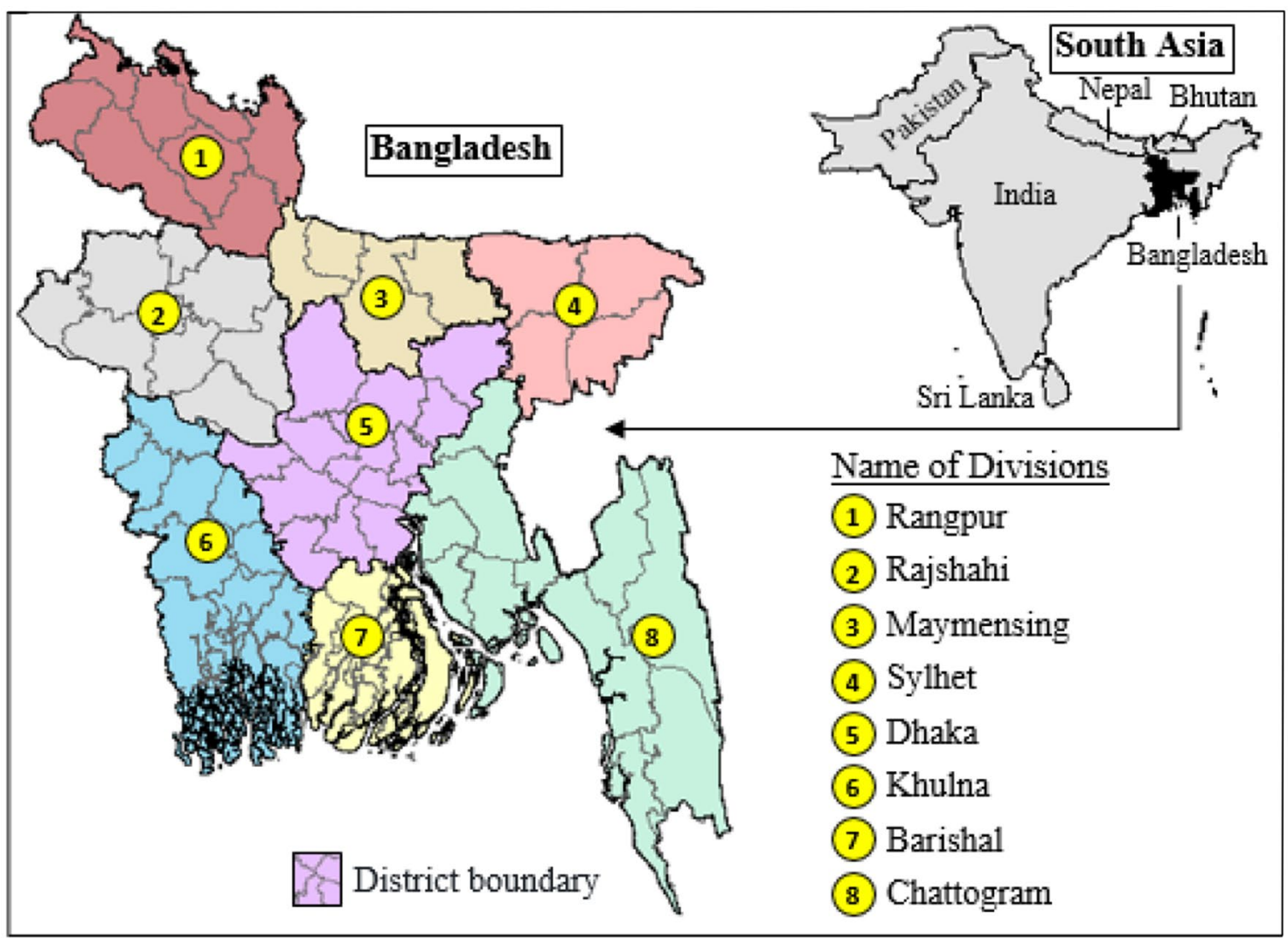

Fig. 1 Study area: Bangladesh is selected as study area and the districts, which are the second level of administrative unit from the top were selected as the spatial units for this study. There are 64 districts

and Chattogram divisions, respectively (BBS 2015a). High population density, urban housing density, location of economic hubs, migrant households, concentration of industry/ factory workers, etc. gear up the spreading of the virus and make the country vulnerable to the disease.

\section{Data used}

The daily time-series COVID-19 data, including confirmed cases, deaths, and recovery from 08 March to 08 August 2020 (154 days), were used here and mainly obtained from the Institute of Epidemiology, Disease Control and Research (IEDCR) website (IEDCR 2000) that updated real-time information on COVID-19 every day by date and district. Two data sets, daily country wise and district wise were collected and analyzed. Some missing data and anomalies in the datasets were verified, adjust and added in the datasets using the information from the daily national and local newspaper and internet. Information regarding interventions taken by government and people movement and activities were collected from the daily press release, Directorate General of Health Services (DGHS), TV news bulletins, and newspaper. It may be mentioned here that the district-wise death in Bangladesh under eight divisions (first level of administrative unit from the top). The divisions are Dhaka, Chattogram, Khulna, Rajshahi, Rangpur, Barishal, Maymensing, and Sylhet

information, IEDCR, has not published regularly; therefore, apart from IEDCR, the available deaths data in the local and national newspapers, local authorities, and offices (District Commissioner office, Civil Surgeon office etc.), were collected and used. These data again were crosschecked with the division-based deaths data which were regularly released by the IEDCR (IEDCR 2020). The age-sexrelated country-level COVID-19 data were collected from the WHO weekly coronavirus situation report, Bangladesh (WHO 2020f). For comparison, data from others countries were used and collected from the COVID-19-related international web portal and dashboard [Worldometers $<$ https ://www.worldometers.info/coronavirus/ $>$, WHO $<$ https:// covid19. who.int/ $>$, Statista $<$ https://www.statista.com $>$, Our World in Data $<$ https://ourworldindata.org/coronaviru $\mathrm{s}>$, IndexMundi $<$ https://www.indexmundi.com/coronaviru $\mathrm{s} />$ ]. Besides, district-wise data for the selected factors were used in the COVID-19 vulnerability zoning, mainly collected from the yearly statistical reports of the Bangladesh Bureau of Statistics (BBS) (BBS 2015a, b, c, d, e, 2019). All population-related datasets were collected for the year 2011-2016; these were the most recent published data that we could obtain. 


\section{Methods}

In the analysis process, Microsoft MS Excel software was used to store and organize the original data and calculate the incident parameters as required in this study. The processed data in Excel were then converted into geographic information system (GIS) data. ArcGIS Desktop software (version 10.7; Environmental Systems Research Institute, Inc) was used to analyze data spatially. In ArcGIS environment, we analyze data spatially for spreading, emerging spatial and temporal hotspot identification, vulnerability zoning, and providing the visualization of the results.

For hotspot analysis, we used Getis-Ord Gi* (G-istar) statistics (Getis and Ord 1992), and Z score, $p$ value, and classification of each bin were calculated and mapped. The resultant $z$ scores and $p$ values tell where features with either high or low values cluster spatially. A high $z$ score and small $p$ value indicate a spatial clustering of high values and vice versa. Thus, this method provides statistically significant hot and cold spots of the incidence. The G-i-star statistics can be calculated as Eq. 1:

$$
G_{i}^{*}=\frac{\sum_{j=1}^{n} w_{i, j} x_{j}-\bar{X} \sum_{j=1}^{n} w_{i, j}}{S \sqrt{\frac{\left[n \sum_{j=1}^{n} w_{i, j}^{2}-\left(\sum_{j=1}^{n} w_{i, j}\right)^{2}\right]}{n-1}},}
$$

where, $x_{j}$ is the attribute value of feature $j, w_{i, j}$ is the spatial weight between feature $i$ and $j, n$ is the equal to the total number of features, and $\bar{X}$ and $S$ calculated as Eqs. 1.1 and 1.2 , respectively:

$\bar{X}=\frac{\sum_{j=1}^{n} x_{j}}{n}$

$S=\sqrt{\frac{\sum_{j=1}^{n} x_{j}^{2}}{n}}-(\bar{X})^{2}$.

Moreover, for vulnerability zoning of COVID-19, analytical hierarchy process (AHP) and weighted sum method (WSM) were used, and an integrated COVID-19 vulnerability zoning index (Cov19VZI) was computed and grouped into high, medium, and low vulnerable zones. In the process, first, relevant factors were selected and spatial distribution of each factor reclassified into five categories-very high, high, medium, low, and very low to standardize the factor. Afterwards, the threshold of the degree of vulnerability for each category of each factor to COVID-19 was established and reclassified. A $0-1$ scale and pairwise comparison technique along with the AHP was used to determine the degree of vulnerability for each category. In this scale, 0 and 1 represent the lowest and highest vulnerability, respectively. Between the values of 0 and 1 , as values increase from 0 , the degree of vulnerability also increases gradually. The AHP is based on pairwise comparisons of elements in a decision hierarchy, and in this study, pairwise comparisons were made using a scale designed by Saaty (1977), which provides ratings on a nine-point continuous scale (Table 1). Details of the AHP method can be found in Saaty (1977), Malczewski (2006), Rahman and Saha $(2007,2008)$, and Rahman et al. (2009, 2014). For assigning the threshold of the degree of vulnerability using the AHP technique, the emphasis was given more in the very high category than high, and again more in the high than medium category, and so on (very high $>$ high $>$ medium $>$ low $>$ very low). After standardization (assigning degree of vulnerability for each category) of all factors, the next step was to establish a set of weights for the factors based on its relative significance to each of the other factors to vulnerability. Here, we also calculated weights for each factor by pairwise comparison and AHP. The weights, which were calculated, are shown in Table 2. Details of the selected factors are discussed in the Results section. For assigning the weights using the AHP technique with expert knowledge, WEIGHT module of IDRISI Andes software was used. Finally, Cov19VZI was computed numerically by a weighted sum method (WSM) for each district. In the WSM, standardized factors multiplied by its corresponding weights and then summed to obtain the vulnerability zoning index of the COVID-19. Therefore, Cov19VZI can be expressed as Eq. 2:

$\operatorname{Cov} 19 \mathrm{VZI}_{i}=\sum_{j=1}^{n} X_{i j} \times W_{j}$,

where Cov19VZI $i$ is the vulnerability zoning index of the district $i, X_{i j}$ is the standardized score of district $i$ of factor $j, W_{j}$ is the weight of factor $j$, and $n$ is the total number of factors. Using Eq. 2, Cov19VAZI ${ }_{i}$ was calculated for each district, and the computed vulnerability area index was standardized using the maximum value method (Eq. 3) to get vulnerability indices that range from 0 to 1 (Rahman et al. 2009, 2014, 2015):

Table 1 Continuous rating scale for pairwise comparison of Saaty's method (Saaty, 1977)

\begin{tabular}{|c|c|c|c|c|c|c|c|c|c|}
\hline \multirow{2}{*}{$\begin{array}{l}\text { Scale } \\
\text { Level }\end{array}$} & \multicolumn{9}{|l|}{ Rating } \\
\hline & $1 / 9$ & $1 / 7$ & $1 / 5$ & $1 / 3$ & 1 & 3 & 5 & 7 & 9 \\
\hline $\begin{array}{l}\text { Level } \\
\text { Impact }\end{array}$ & $\begin{array}{l}\text { Extremely } \\
\text { Less impo }\end{array}$ & Less important & Strongly & Moderately & $\begin{array}{l}\text { Equally } \\
\text { Equal }\end{array}$ & $\begin{array}{l}\text { Moderately } \\
\text { More impor }\end{array}$ & $\begin{array}{l}\text { Strongly } \\
\mathrm{t}\end{array}$ & Very strongly & Extremely \\
\hline
\end{tabular}


Table 2 weights by AHP for each category of each factors, and factors weight individually

\begin{tabular}{|c|c|c|c|c|c|}
\hline \multicolumn{2}{|c|}{$\begin{array}{l}\text { Standardized weight } \\
\text { (Scale } 0-1)\end{array}$} & \multicolumn{4}{|l|}{ Factors weight (scale $0-10$ ) } \\
\hline Category & Weight & Factors & Weight & Factors & Weight \\
\hline Very high & 0.37 & 1. Urban population & 2.10 & 7. Industry worker & 0.50 \\
\hline High & 0.23 & 2. Urban male population & 1.90 & 8. Population density & 0.30 \\
\hline Medium & 0.17 & 3. Establishments employee & 1.60 & 9. Floating population & 0.20 \\
\hline Low & 0.13 & 4. Male population & 1.20 & 10. Working population & 0.20 \\
\hline \multirow[t]{2}{*}{ Very Low } & 0.10 & 5. Number of migrants & 1.00 & 11. Senior citizen & 0.20 \\
\hline & & 6. Household consumption & 0.70 & 12. Literacy rate & 0.10 \\
\hline \multicolumn{2}{|c|}{ Consistency ratio $=0.06$} & Consistency ratio $=0.06$ & & & \\
\hline
\end{tabular}

$\operatorname{Cov} 19 \mathrm{VZI} \mathrm{I}_{\mathrm{si}}=\operatorname{Cov} 19 \mathrm{VZI} \mathrm{I}_{\mathrm{i}} / \operatorname{Cov} 19 \mathrm{VZI} \mathrm{I}_{\max }$

where Cov19VHI ${ }_{s i}$ is the standardized value of Cov19VHI of the district $i$, and $\operatorname{Cov} 19 \mathrm{VHI}_{\max }$ is the maximum value of Cov19VAZI among the district. In the standardized index, high values represent a high vulnerability, and in contrast, low values represent the low vulnerability for COVID19. Finally, to a meaningful zonation of vulnerable area, obtained vulnerability zoning index was graded into three levels of vulnerable areas defined as high, medium, and low.

\section{Results and discussion}

\section{COVID-19: outbreak, interventions, transmission, fatality, and recovery (154 days)}

\section{Outbreak, interventions, and transmission}

Challenges of the outbreak of COVID-19 are not only the virology and epidemiological-related issue but also encompasses with society, economy, and the environment of a particular territory. Hence, a special attention needs to be paid and detail analysis of the nature of the disease is utmost needed to control the spread of the virus and to formulate comprehensive planning against COVID-19. The outbreak of COVID-19 was first reported on 8 March 2020 and three cases were identified in the country (DGHS 2020). Out of three confirmed cases, two of them were returning Bangladeshi emigrant (non-resident Bangladeshi) from Italy and the rest was a female member of the emigrant family returning to Italy. Later, on 15 March 2020, two more cases were identified. One of them was again from Italy and the other from Germany (DGHS 2020). According to the information provided by the IEDCR on 15 March 2020, hundreds of emigrant Bangladeshi have arrived in Bangladesh from Italy by this time. However, a large part of them has observed the home quarantine and institutional quarantine as administration decision (DGHS 2020). On 16 March 2020, three more new cases were recorded and they all were the family members of one of the patients identified on 15 March 2020 (DGHS 2020). Just next day, i.e., on 17 March 2020, two more new cases were added in the infected list and, surprisingly, one of them was returning emigrant from Italy and was in quarantine. The other one was in contact with a person with symptoms of COVID-19 from USA. However, the USA citizen returned to the USA without any contact with the COVID-19 control room in Bangladesh (DGHS 2020). Moreover, all those who were infected with COVID19 while living in the country other than returning Bangladeshi emigrants in the initial days of the outbreak, they were either relatives or with the contact of those infected returning emigrants (DGHS 2020). Thus, based on the information outbreak and nature of spreading of COVID-19 in Bangladesh in early days, it may be said that the COVID19 outbreak and initial transmission has taken place in the country by the returning Bangladeshi emigrants, and first two returned emigrants from Italy on 8 March 2020 can be considered as patient 0 (zero) for Bangladesh. Besides confirmed cases, afterwards, it was observed that infections remained low until the end of March (reported 51 confirmed cases and five deaths on $31 \mathrm{March}$ ), but saw a steep rise in April and later, and the number of infections in the country has grown significantly (IEDCR 2020).

Despite taking initiatives before the first case identification in the country, on 14 March, the Bangladesh government suspended on arrival visas for all nationalities and on 16 March 2020 closed all educational institutes. Later, the country declared the general holidays as lockdown from 26 March to 4 April 2020 to contain the spreading of COVID19. The lockdown has extended several times until 30 May 2020 (DGHS 2020). The major interventions that were taken by the government to prevent the spreading of the virus and the activities followed by the mass people are highlighted in Fig. 2 as the major timeline for COVID-19 outbreak in the country. One of the main objectives of the study was to analyze the nature of the spreading of COVID-19 over the stipulated time. The analysis of the 154 days data depicts that in the initial days of the outbreak, the number of affected people by COVID-19 remained very low and a total number 


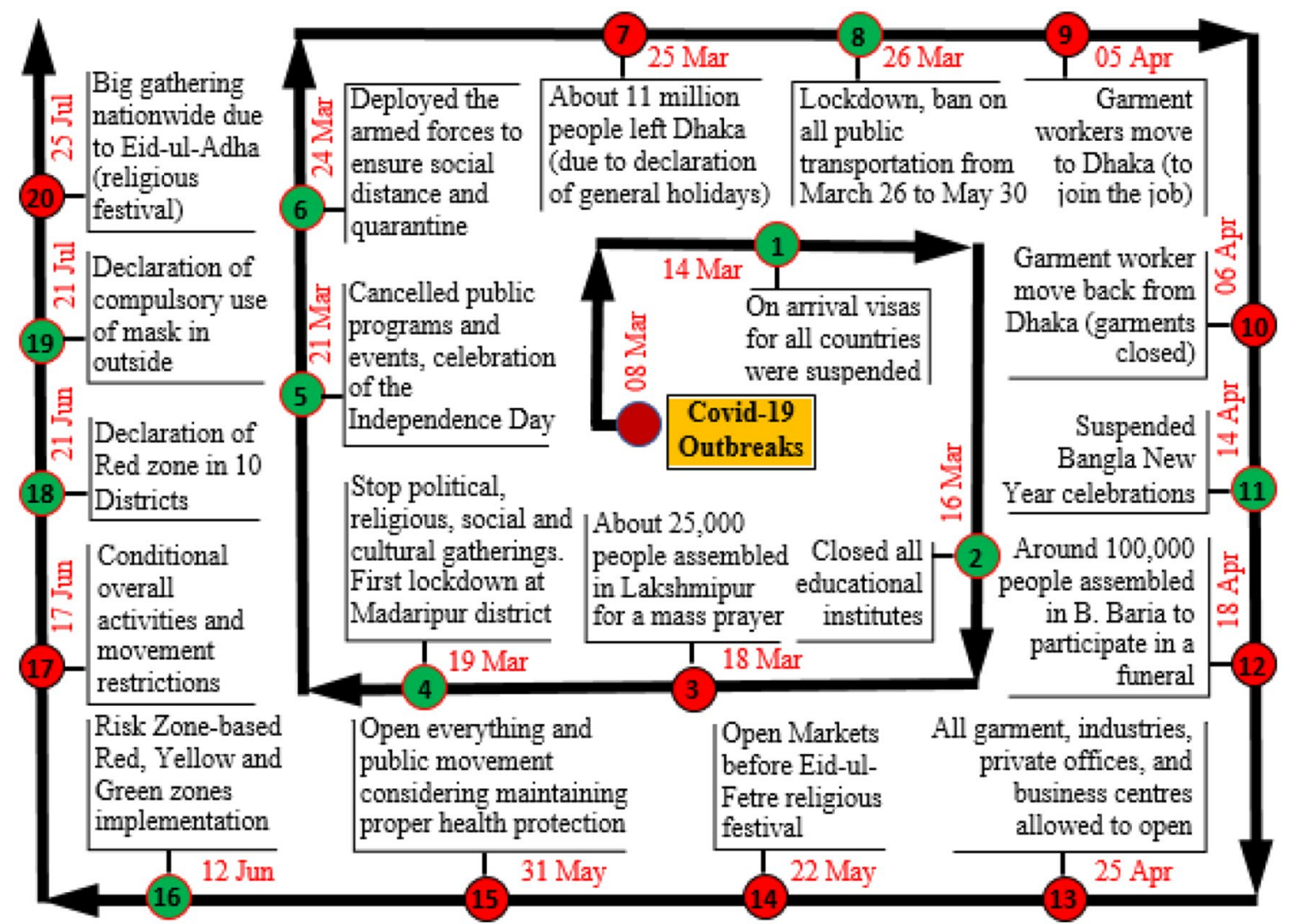

Fig. 2 Timeline of major interventions/activities (green and red circles represent positive and negative interventions/activities, respectively). Activities indicated in the green circles act to control the

of confirmed cases exceeded 100 (123 total cases) on 6 April 2020 at the day 30 of the virus outbreak. However, infections increased sharply afterwards in both the circumstances, daily and total confirmed cases (Table 3 ). The total cases of COVID-19 touched the number 1000 on 14 April 2020 (1012 total cases at day 38), and a recorded of 209 infected individuals in a single day. Again, with a new hit of 688 cases in a single day, total cases reached 10,000 scale on spreading of the disease, and activities mentioned in the red circles act to spread the disease

04 May $2020(10,143$ total cases at the day 58) [Table 3]. Furthermore, on 02 June 2020 at outbreak day 87, total cases crossed 50,000 scale (recorded 52,455 total cases) with a 2911 daily infected persons. Surprisingly, just after 16 days (at the day 103), i.e., on 18 June 2020, the total cases jumped over 100,000 scale and recorded 102,292 total cases with 3803 daily cases. It may be mentioned here that on 13 June 2020, considering the total confirmed cases, Bangladesh was

Table 3 Transmission of COVID-19 at different level of scale

\begin{tabular}{|c|c|c|c|c|c|c|c|}
\hline Day of outbreak & Date & $\begin{array}{l}\text { Time taken } \\
\text { (days) }\end{array}$ & Level of scale & $\begin{array}{l}\text { Total con- } \\
\text { formed cases }\end{array}$ & $\begin{array}{l}\text { Daily confirmed } \\
\text { cases }\end{array}$ & Daily death & Total death \\
\hline Day 1 & 08 March & 0 & 1 & 3 & 3 & 0 & 0 \\
\hline Day 30 & 06 April & 29 & 100 & 123 & 35 & 3 & 12 \\
\hline Day 38 & 14 April & 08 & 1000 & 1012 & 209 & 7 & 46 \\
\hline Day 58 & 04 May & 20 & 10,000 & 10,143 & 688 & 5 & 182 \\
\hline Day 87 & 02 June & 29 & 50,000 & 52,445 & 2911 & 37 & 709 \\
\hline Day 103 & 18 June & 16 & 100,000 & 102,292 & 3803 & 38 & 1343 \\
\hline Day 117 & 02 July & 14 & 150,000 & 153,277 & 4019 & 38 & 1926 \\
\hline Day 133 & 18 July & 16 & 200,000 & 202,066 & 2709 & 34 & 2581 \\
\hline Day 153 & 07 August & 20 & 250,000 & 252,502 & 2851 & 27 & 3333 \\
\hline
\end{tabular}


ahead of China, the Zero ( 0 ) ground country of the virus outbreak (Penerliev and Petkov 2020). The situation becomes further worsen on 02 July 2020 (at day 117) and the total cases crossed 150,000 within 14 days only (Table 3). Later, on 07 July 2020 (at day 122), the total number of COVID19 cases of Bangladesh (168,645 total cases) exceeded the total number of cases of France (168,335 total cases) [DGHS 2020; IndexMundi 2020]. Then again, on 18 July and 07 August 2020, total infections crossed the bar of 200,000 and 250,000 and recorded 202,066 and 252,502 cases, respectively (Table 3 ). Thus, the transmission of COVID19 depicts that rapid rise in the number of confirmed cases was observed from May and onward (Table 3, Fig. 4). On the other hand, though there was a very limited number of test for COVID-19 at the beginning, however, the number of tests was increased gradually (Fig. 3), and as of 29 August 2020 , total 1,525,815 tests were conducted which amounts to 9250 tests per million people. The country's rank was 30th in the most impacted countries worldwide according to the rate of COVID-19 tests performed per million population, and as of 29 August 2020, in South Asia, Bangladesh position was just behind the position of India (28,563/million) and Pakistan (11,552/million) [Statista 2020]. This was mainly because of the insufficient PCR machines and kits, biosafety labs, and skilled health workers and technicians (Sakamoto 2020).

It is a fact that NPIs, such as lockdown, social distancing, isolation, quarantine, restricting public events, etc., are followed globally to control the spread of COVID19 , since there is no effective medication available. The impact of NPIs which were taken time to time in the country can be analyzed viewing the timeline of interventions implementation and the daily number of confirmed cases and the rate of daily confirmed cases. Figure 4 depicts that the implementation of necessary steps at the beginning of the outbreak, the number of infection, and rate of infection were very low till mid of April. However, from the mid-April to the end of April, the rate of confirmed cases was a little bit high and increased gradually soon after. On 06 May 2020, coronavirus outbreak spreads over to all 64 districts of Bangladesh (DGHS 2020). This widespread of the infection may be correlated with the unexpected movement and activities of the mass people, like the movement of millions of people to home districts from Dhaka on 25 and 26 March owing to the declaration of general holydays that aided to spread the disease nationwide, movement of garment workers to Dhaka from different parts on 05 April to join the workplace and again a force to move back from
Fig. 3 Daily confirmed cases verses daily test conducted. $X$-axis represents time (day) and $y$-axis represents number

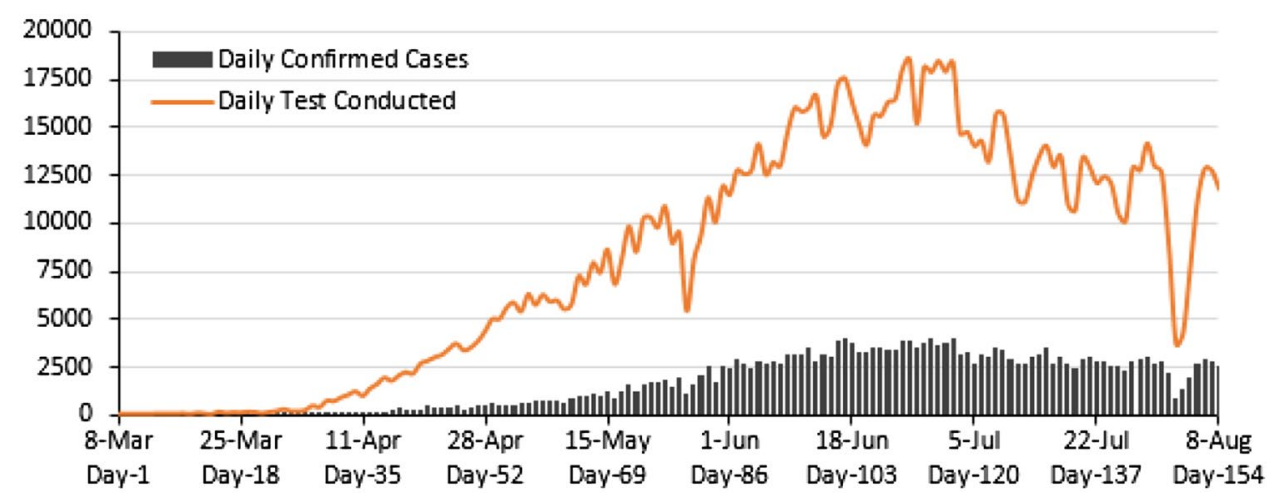

Fig. 4 Daily confirmed cases (number) and rate of daily confirmed cases against daily test conducted. $X$-axis represents time (day), and $y 1$ and $y 2$ axes represent number for daily cases and $\%$ of the rate of cases. Vertical green and red broken lines represent the timeline of major positive and negative interventions/activities, respectively. For details for vertical line (interventions/activities) descriptions, see Fig. 2

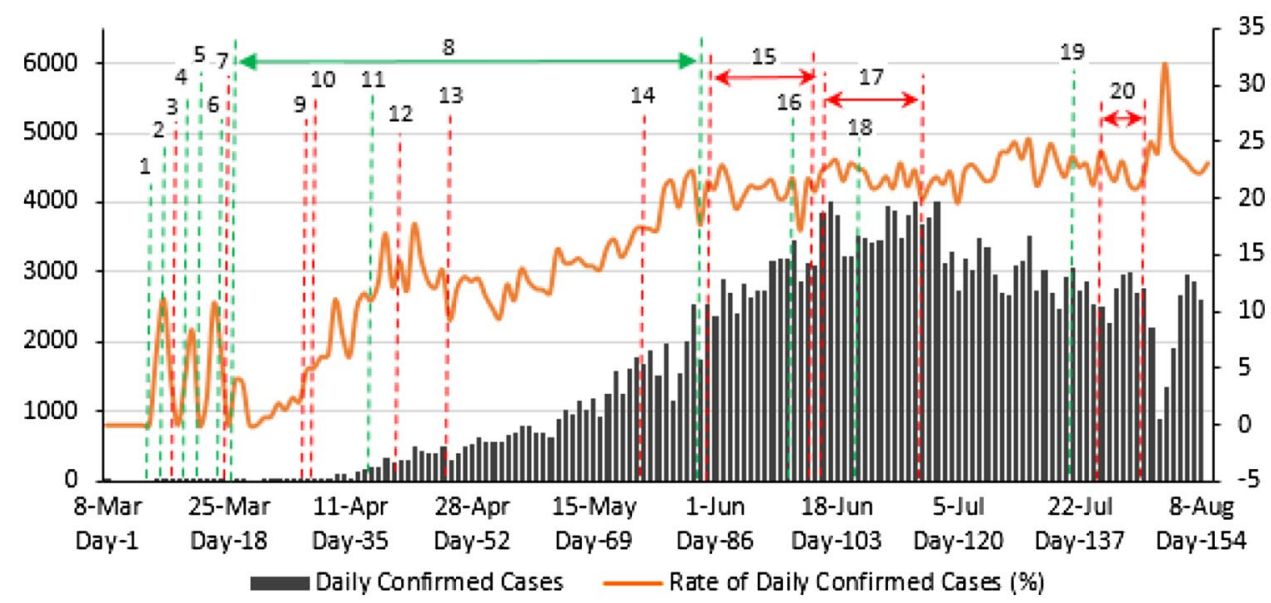


Dhaka on 06 April 2020 due to the shutdown of all garment industries (Fig. 2). Again, Fig. 4 illustrates that the rate of infection was comparatively low in the lockdown days (from 26 March to 30 May 2020). Therefore, it may be noted here that though the government has taken some effective measures to minimize the spreading of COVID19 , ignoring to maintain the social distance, government's advice and proper health safety measures by the citizen worsen the situation in later days. Furthermore, there was a decreasing trend was observed in the number of daily confirmed cases from 3 July 2020, and apparently, we can understand that this may be the starting of a downward trend of infarction (Figs. 3 and 4); however, if we look at the number of daily tests conducted in Fig. 3, it would be clear that the downtrend of infection was mainly the result of less number of daily tests conducted compared to the previous days. Thus, there was inconsistency in the tests conducted daily, and hence, to explain the COVID19 situation based on only the distribution of daily cases over time would not be practical. As therefore, time-series plot of the rate of daily and total confirmed cases against the daily and total test conducted, and their growth factors were analyzed. As shown in Fig. 5, the rate of both daily and total cases was comparatively low in the early days of spreading of COVID-19, but in the later days, both were increased continuously. The daily infection rate against the number of daily tests conducted was more than 20 (20 out of 100 people) from the 25 May 2020 and reached height $31.91 \%$ on 03 August 2020, which denoted that the rate of infection was in rising trend (Fig. 5), and during the study period, transmission presents an increasing trend. The continuous increasing rate of both daily and total cases also indicated the severity of the transmission of the virus in these days. Moreover, the growth factor (GF), i.e., change of daily, and total infection rates (rate of particular day/rate of the previous day) were also analyzed. The GF value $<1,1,>1$, and persistently $>1$ indicate a decline, which remains the same, increase and exponential increase of the change or growth, respectively. Highest but uneven change or growth in both daily and total infection rates were observed in the beginning days of COVID-19 cases in Bangladesh (Fig. 5). Though there were up (increased) and down (declined) in daily infection rate during the time, however, since 3 May 2020 (day-57), the GF of total infection rates were within 1.00-1.02, indicating that the attack rate of the virus was mostly in same or greater magnitudes during the time (Fig. 5) and the same trend of infection continues. Thus, a special attention and interventions are required to minimize the spreading of the virus.

Furthermore, the analysis of the doubling time of the confirmed cases over time is important to know how quickly the number of cases increased. For instant, Bangladesh recognized 123 cases on 06 April 2020 and 330 cases just after 3 days on 9 April 200, means that it took 3 days to double (cases doubling time). In Fig. 6, background broken grey lines are the models for the paths of doubling times 1, 2, 3, 4, 5 days and so on. If the cases distribution line is steeper than a particular model grey line, then doubling time of the cases will be faster than the model line. As seen in Fig. 6, in the beginning, the doubling time of infection spreading counted double faster than every 3 days, and till the end of April, the doubling time of infection spreading was faster than every 4 days. Nonetheless, from 30 April 2020, the doubling time of the number of confirmed cases began to decline gradually, and as of 08 August 2020, the overall case doubling time was 11 days. Figure 6 further gives a comparative picture of the increase of infection between Bangladesh and among some selected countries of South Asia, South East Asia, and Europe. The figure depicts that the disease in most countries develops a common and faster logarithmic upward trend during the first 15-20 days after that the first 100 cases identified. However, in the days to come infection continued to increase but at comparatively slower rate. The figure further shows that Bangladesh observed much higher increasing trend and faster doubling time compare to the cases of Sri Lanka, Malaysia, Indonesia, and Pakistan. Even as of 08 August, the doubling time was faster than China, Germany, and France and almost same as Italy and the UK, denoting the severity of the infection.
Fig. 5 Rate ( $y 1$-axis in \%) and growth factor (change) [ $y 2$-axis in unit] of daily and total confirmed cases (infection)

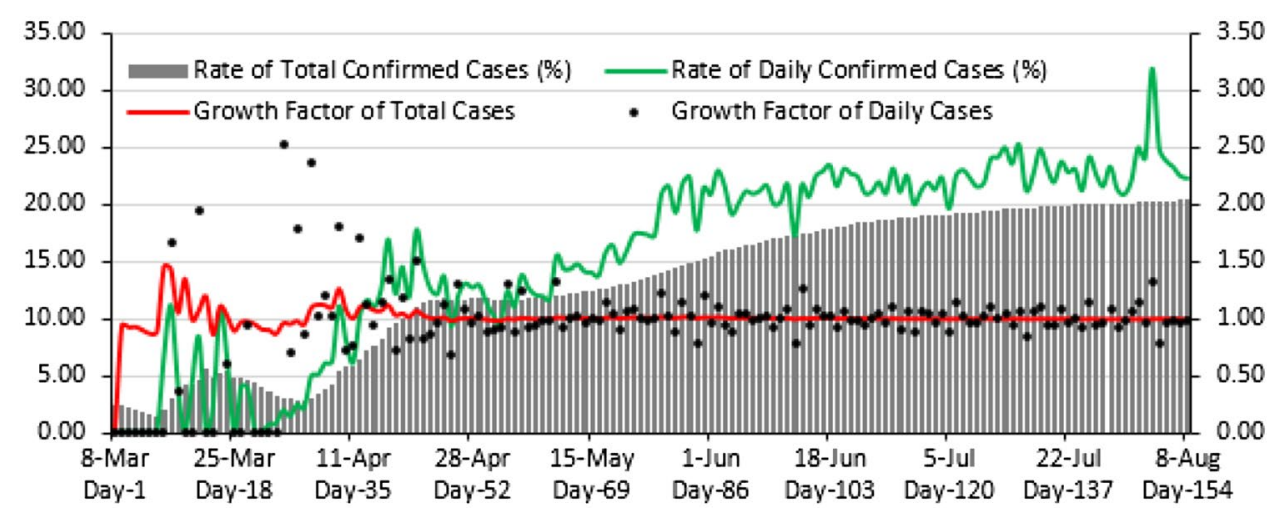


Fig. 6 Doubling time of the growth of COVID-19 confirmed cases in Bangladesh and some other selected countries starting from the day of 100 confirmed cases. Broken grey lines (d1d60) represent the models for the paths of doubling times. $X$-axis represents days (time) and $y$-axis represents cumulative number of confirmed cases at logarithmic scale

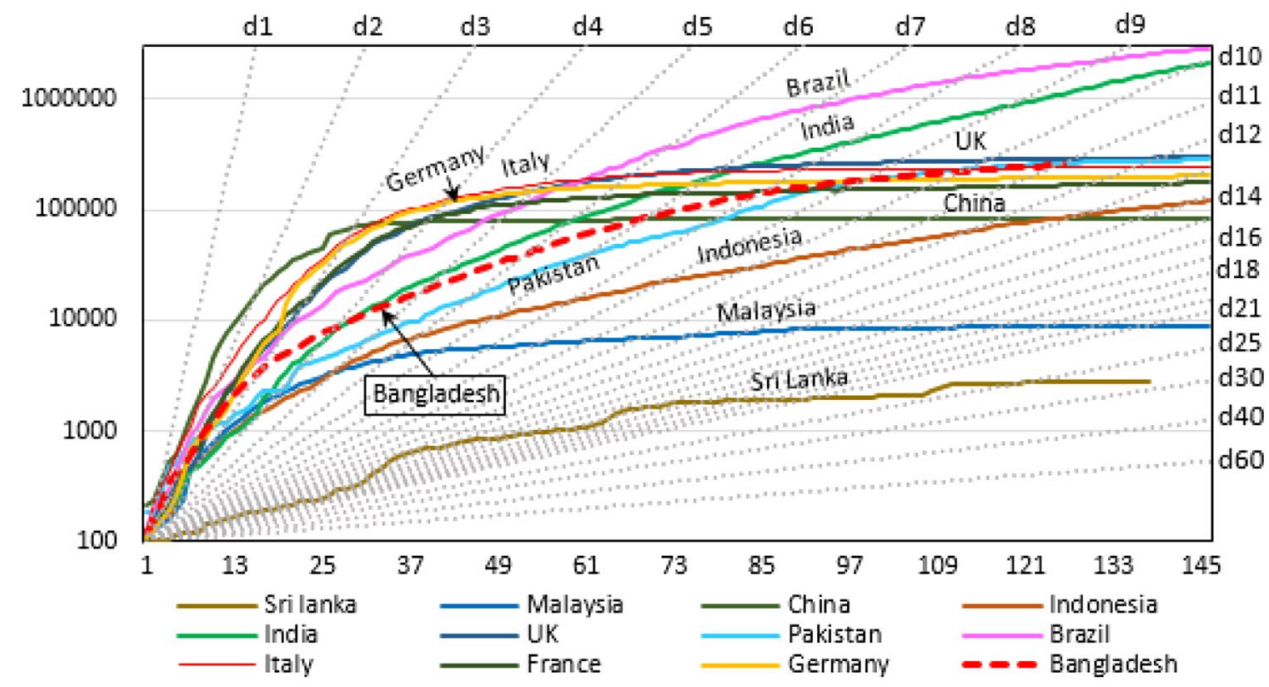

\section{Fatality}

The death toll or fatality of any disaster is an important concern and the severity of the incident is intended by the number of deaths. In case of the pandemic COVID-19, as of 29 August 2020, there were 843,165 deaths recorded worldwide, and Bangladesh ranked was 29th in fatality with 4206 deaths (25 deaths per million people) (Worldometer 2020). Bangladesh reported its first coronavirus death on 18 March 2020. (IEDCR 2020). In this study, we consider the analysis of fatalities as daily deaths and fatality rate over time. The fatality rate is the number of deaths outcomes as a percentage of the total number of confirmed cases (Penerliev and Petkov 2020). This measure reflects the severity of a disease in a specific time. In Fig. 7, it can be seen that the daily death toll was below 10 up to 6 May 2020, but a sharp increase of daily deaths was observed thereafter. The highest deaths in a single day were recorded 64 on 30 June 2020. Conversely, although the death toll was low in the beginning, the fatality rate was comparatively high, and the highest single-day fatality rate was recorded 12.82 (12.82 deaths out of 100 infected persons) on 25 March 2020 (Fig. 7). However, as seen in Fig. 7, from 17 April 2020, the fatality rate began to decline gradually and the rate was between 1.28 and 1.32 from 01 June to 08 August 2020, denoting the fatality rate remained almost same during the period. In a developing country like Bangladesh, this is actually an alarming situation and to reduce the fatality due to COVID-19, health, and medical infrastructure along with highly improved healthcare system need to be developed. Since prevention is better than cure, the spreading of COVID-19 also needs to be controlled by taking appropriate measures. Although the fatality rate was far below in Bangladesh (only 1.35\%, as of 28 August 2020) compared to the global rate (3.37\%) and rates in the highest recorded countries, Yemen (29.07\%), Italy (13.44\%), UK (12.55\%), Belgium (11.84\%), Hungary (11.41\%), and even South Asian countries Pakistan (2.13\%) and India (1.82\%) (Statista 2020), the country needs to be prepared as if the situation does not deteriorate further.

In addition, confirmed cases and deaths analysis based on demographic aspect reveal that there was a strong demographic variation related to the age-sex group in COVID-19 pandemic. As seen in Fig. 8, compared to the male-female ratio, there was a considerably higher proportion of cases
Fig. 7 Daily death and fatality rate. $X$-axis represents time (day), $y 1$-axis represents daily death in number, and $y 2$-axis represents fatality rate in $\%$

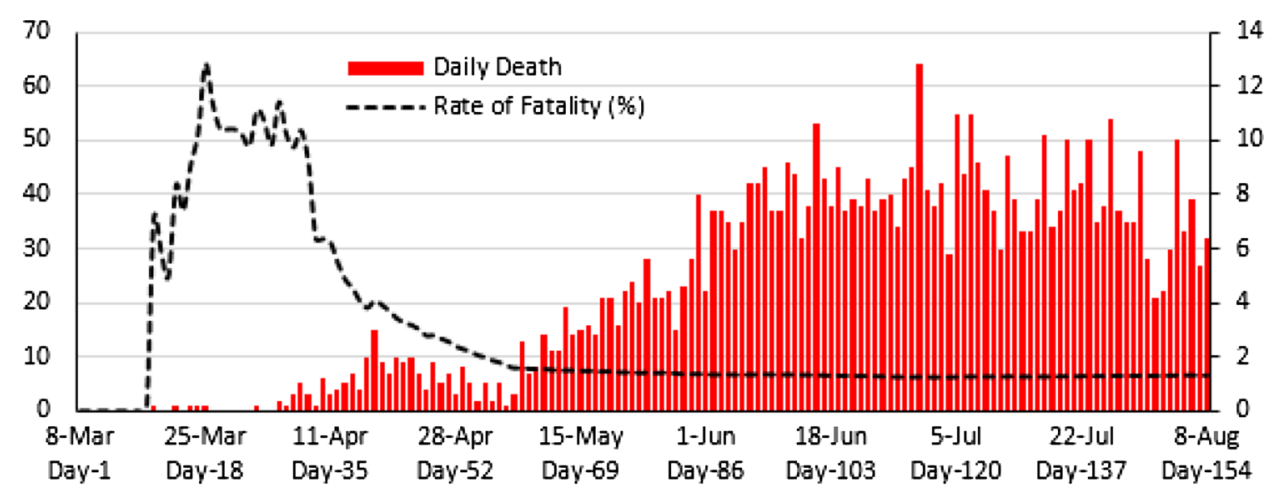


Fig. 8 Age-sex composition of COVID-19 (as of 20 July 2020): a reported cases and $\mathbf{b}$ deaths. $X$-axis represents \% of the total number of cases/deaths and $y$-axis represents age group. Shaded portion represents most vulnerable groups

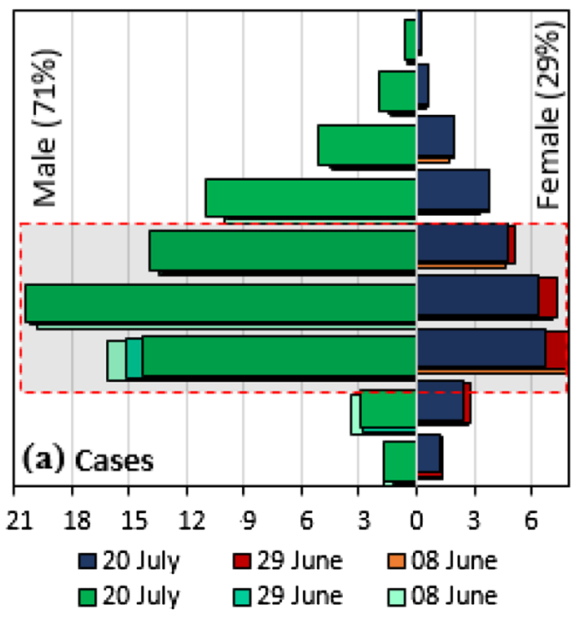

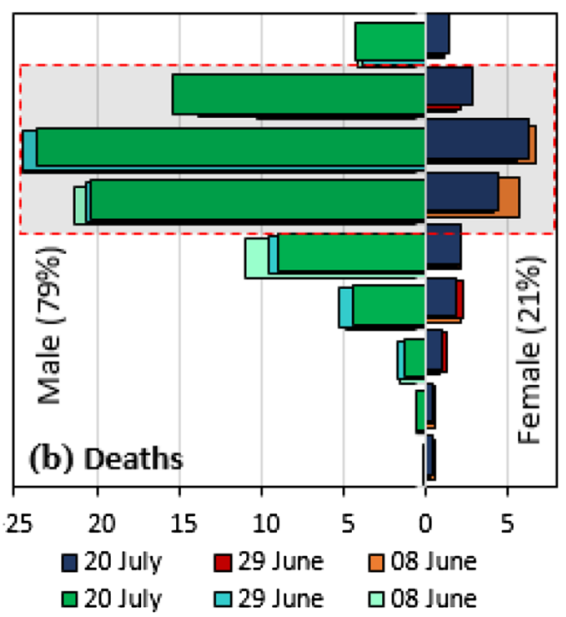

and deaths among the male population than female in all the stipulated times, i.e., 8 June, 29 June, and 20 July 2020. As of 20 July 2020, male contributed to $71 \%$ among the confirmed cases and, in contrast, female contributed to $29 \%$ only among the reported cases [Fig. 8a]. Although in some different age-sex groups, there was a slight variation in percentage distribution of cases in the different time period, as of 20 July 2020, the infection rate was the highest among those aged 21-50 years for both male (48.5\%) and female (18.3\%). The highest confirmed cases for the male population were among the age grouped 31-40 (27.6\%), followed by $21-30(14.4 \%)$ and $41-50(13.8 \%)$ age grouped. In contrast, the highest cases were identified for female among the age grouped $21-30(6.9 \%)$, followed by age group 31-40 (6.6\%) [Fig. 8a]. Again, as seen in Fig. 8b, as of 20 July 2020, of the total deaths, $79 \%$ and $21 \%$ were male and female, respectively. The fatal outcome was the highest among both the male and female patients aged-grouped 61-70 years (male $23.7 \%$, female $6.1 \%$ ), followed by those aged-grouped 51-60 (male $20.3 \%$, female 4,4\%) and 71-80 (male $15.1 \%$ and female $2.8 \%$ ). Among patients aged 40 and below, the death toll was significantly lower, $4.6 \%$ and $1.4 \%$ for male and $2.0 \%$ and $1.2 \%$ for female for the age-groups $31-40$ and $21-30$, respectively. It was even lower, below $1 \%$ among patients aged 20 or younger for both genders (Fig. 8b). Therefore, the infection rate was more among the male and working-age people, while the fatality rate was more among the male and above-working age (age 60 and above) people. The finding is broadly consistent with the global findings, particularly for the fatal cases for aged people (60 and above) (Penerliev and Petkov 2020; Chan et al. 2020; Bergquist and Rinaldi 2020; Wu and McGoogan 2020). Hence, in Bangladesh, younger male people are more vulnerable to get the infection and senior male citizens more prone to fatal than female. From a demographic point of view, age group and gender can be treated as important vulnerable factors for COVID-19 consequences. In Bangladesh, for social or cultural reasons usually, men do more outdoor activities than women, and women are some extent dependent on male family members, and they are introverted too. Moreover, the working-age male population are more active for outdoor activities than female. This may be one of the main reasons for having a higher rate of infection for male. However, the reasons for this sex difference remain unclear, and it was suggested that due to the defense of $\mathrm{X}$ chromosomes and sex hormones which play a major role in immunity for women could be a factor for women's reduced vulnerability to viral infections (Zhu et al. 2020b; Voinsky 2020). Again, a higher rate of male infection could reflect a relaxed social distancing, avoiding the use of protective kits, overconfidence, and ignoring severity of the disease among the young male population than female. Therefore, to prevent the spreading of virus and reduce the fatality, preventive measures like social/physical distancing, temporal separation, movement restrictions, use of protective kits (mask, hand gloves, and sanitizer), and awareness development in these vulnerable groups should be implemented as current preventive measures. Most countries in the world particularly, the countries of high and very high human development group, have widely applied NPIs to slow the transmission of the COVID-19 (UNDP 2020).

\section{Recovery}

In any pandemic, the recovery from the infection is important in a sense of overcoming the crisis and generating natural immunity among the people. As of 29 August, there were 25,027,198 confirmed cases identified worldwide and among those 17,388,940 cases were recovered. Bangladesh ranked was 17 th based on people recovered from the pandemic COVID-19 (recovered 198,863 patients, as of 29 August 2020). The USA, Brazil, and India were among the top three listed countries according to the number of recoveries (Worldometer 2020). Here, we analyze the total number 
of recovery and rate of recovery (the number of recovery outcomes as a percentage of the total number of confirmed cases) over time. From the data analysis of recovery in Bangladesh, as seen in Fig. 9, it can be noted here that, until 13 April 2020, the total number of recoveries of reported cases was very low but higher than the cumulative death. Afterwards, the number of recoveries increased gradually, and from 15 June 2020, an accelerating number of recoveries was recorded. This somehow indicates that natural immunity is taking place among the people to COVID-19, and also the patient's ability to adapt to COVID-19 infection is increased. Again, as it is highlighted in Fig. 9, the recovery rate was always higher compared to the fatality rate except for 12 April to 02 May 2020 . The recovery rate was only $02-06 \%$ which was almost same as the fatality rate at this time. However, after that the recovery rate increased rapidly and on 29 August 2020, the recovery rate reached at 62 (about 62 out of 100 cases) which was quite close to the global average recovery rate $(69.48 \%)$, even it was pretty higher than the USA (55.32\%), the 1st ranked country according to the number of confirmed cases (Statista 2020). However, the rate was much lower than Brazil (78.08\%), India (76.59\%), and Russia (81.63\%) (calculate using data from Worldometer 2020). The recovery of the patients mainly depends on the availability of the effective health system, immunity level, and mental health of the patients and the rules and regulation which needs to be followed by the patients of
COVID-19. Thus, to recover more, it is necessary to develop effective health system, and on the other hand, it is also required to take needful steps to boost up the immunity level and improvement the mental health of the patients, and also patients need to be followed the rules and regulations which are recommended by the authority and the health experts.

Again, to get the clearer picture of the pandemic, we analyzed the circumstances based on epidemiological weeks, since it was stated that the incidence varied greatly by weeks of disease onset and can be effectively examined accordingly (Chan et al. 2020). Figure 10 portraits that in Bangladesh, the number of tests conducted and the number of confirmed cases were increased in every week gradually until the week 17 , total 119,976 tests were conducted, and 25,701 cases were confirmed in this week which was the highest recorded statistics for both the incidences. However, in weeks 18-20, the test conducted and case confirmed both were decreased. Increasing the number of tests conducted gradually over time indicates the ability to strengthening the capacity of the testing facilities in the country. In the beginning, IEDCR lab was the only dedicated laboratory for COVID-19 testing; later, the number of laboratories for COVID-19 testing has increased gradually, and as of 29 August 2020, there were total 92 testing labs functioning all over the country, and the ratio of total testing reached 9250 per million people (IEDCR 2020). Yet, this ratio is still not enough to get a real picture of the pandemic in a country more than 160 million
Fig. 9 Total recovery, total deaths (in number, $y 2$-axis), recovery rate, and fatality rate (in $\%, y 1$-axis) by dates. $X$-axis represents time (day)

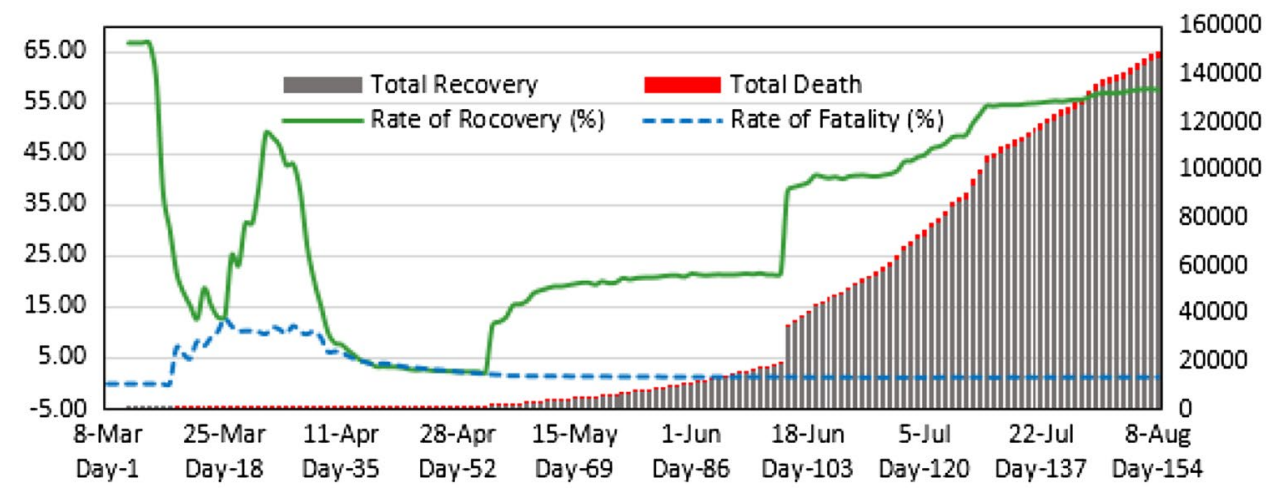

Fig. 10 Epidemiological weekly distribution of test conducted, confirmed cases, recovery (number, $y 2$-axis) and the rates of confirmed cases, deaths, and recovery (in $\%, y$-axis) by week $(x$-axis)

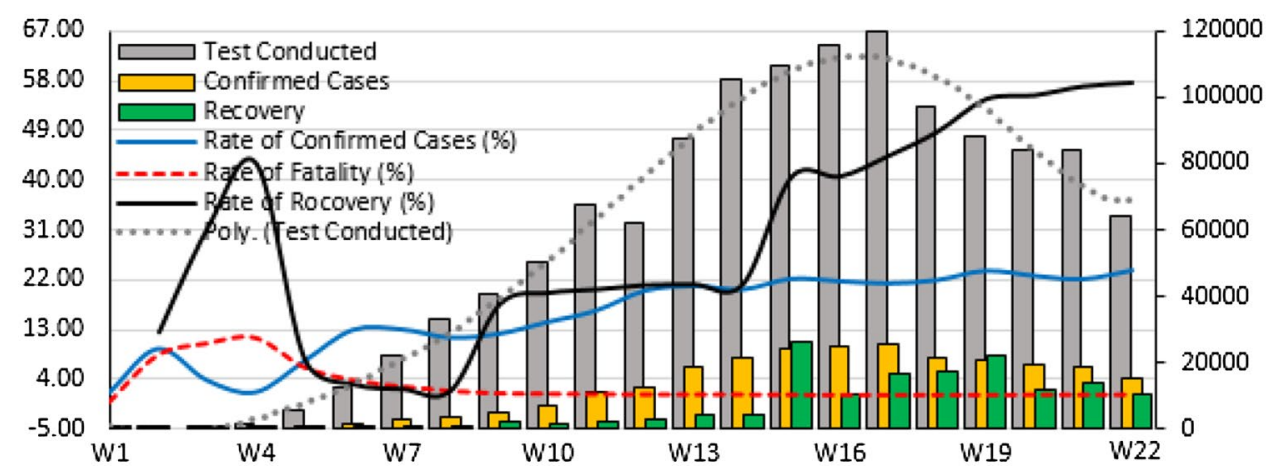


inhabitants. Moreover, the analysis of Fig. 10 shows that as the number of tests increased, the number of infections increased and vice versa. Thus, increasing testing coverage will make the data of positive cases more reliable. Furthermore, although the rate of weekly infection was comparatively less during week $1-11$, the rate was more than $20 \%$ in the later weeks and the highest rate $(23.81 \%)$ was recorded in week 22. This is really an alarming situation, since the condition was uncontrolled and people were getting infected with a high rate for a long. On the other hand, weekly fatality statistics depicts that the number of deaths increased up to week 18 except weeks $8,9,15$, and 16 . The highest 308 deaths were recorded in week 18 . Nevertheless, the deaths again decreased in weeks 19 and 20 (Table 4). And, in comparison to the early weeks particularly, weeks from 1 to 5 , the fatality rate of COVID-19-related death was higher than the later weeks and from week 6 and onward, the fatality rate began to decline. However, from weeks 11 to 21 , the fatality rate varies between 1.3 and $1.4 \%$, denoting that there was no improvement in the control of deaths situation for the last 11 weeks. Besides, the number of recovery by weeks highlighted that the number of patient's recovery was increased remarkably from the week 15 . The recovery rate reached to $57.47 \%$ in the week 19 . This is a positive sign and people are getting recovered almost closed to the global average rate of recovery (69.48\% as of 29 August 2020). It is also favourable that the rate of recovery was always higher than that of the rates of attack (confirmed cases) and fatality from the week 9 and ahead (Fig. 10).

\section{COVID-19: spatio-temporal pattern and hot spots (154 days)}

\section{Spatiotemporal pattern: reported confirmed COVID-19 cases}

"Everything is related to everything else, but near things are more related than distant things"- the first law of Geography (Tobler 2020) highlights the significance of the space in the study of any phenomenon of the Earth, like COVID-19. The geospatial analysis helps to analyze the spatial dependence and explain the direction and intensity of the spreading of disease on the space as well as in time. Here, we analyzed the infected cases and related deaths due to COVID-19 spatially to get the spatial pattern and the nature of spreading of the disease. Sixty-four (64) districts were selected as the spatial units of analysis (Fig. 1). In the country, the first three confirmed cases were identified on 8 March 2020 in two districts, namely Madaripur and Narayanganj of Dhaka Division (Table 5). On 15 March 2020, the first cases were identified in Dhaka, the capital and Gazipur districts. In March 2020, the first cases were identified in only seven districts. However, the transmission of the virus spread out widely by April 2020, and the first cases were identified in 55 new districts out of 64. By identifying the first patient in Rangamati on 07 May 2020, the virus was spread to all districts of Bangladesh. Complementarily to Table 5, results on the temporal and spatial evolution of the disease dynamics were further presented in Fig. 11. The figure depicted that the disease propagates from the central parts of the country initially by three (3) infectious individuals. Afterwards, in week 2 of the outbreak of disease, infection transmitted to the capital Dhaka and some surrounding districts within $50-100 \mathrm{~km}$. Also, in this week, the infection spread to new two separate locations (Gaibandha and Chuadanga), even though they are relatively far geographically (about $200-250 \mathrm{~km}$ from the capital). These two districts located in north-western and eastern parts of the country, respectively (Fig. 11b). In week 3 , infection diffused to new two south-eastern districts, namely Chattogram and Cox' Bazar. Hence, the spatial spread of the disease signals that initially there were mainly four separate locations which act as prime locations to further spread the infection, and the infection was mainly transmitted by the imported cases and the movement of infected people to those areas or somehow close contact of the people with the infected people of those locations. In week 4, the situation remained almost the same; very few were infected in those locations. However, the distribution and spatial pattern of the cases in week 5 indicates that the number of infection increased remarkably compared to the week 4 (about 20 times higher, Table 4), which was an indication of speed up the spatial dynamics of the disease and starting the community transmission as well. Later, spreading and intensity of the infected cases were mainly concentrated in Dhaka and its adjacent districts (within $100 \mathrm{~km}$ ) up to week 10. Chattogram and Cox's Bazar, the two south-eastern districts came up as second intensified areas of infected cases in weeks 11 and 12. It is worth mentioning here that until week 12 , the number of infected cases was limited, and concentrated in some particular locations, mainly because of the lockdown and other interventions which have taken by the Government during this time. Figure 11 further highlighted that in week 13 to week 22, the transmission of the disease widely and quickly took place in the central, south-eastern, north-eastern, and some north-western and south-western districts with high intensity. Withdrawing the lockdown, relaxing in restrictions of the people movement, lack of awareness, reluctance to follow the rules and regulations etc. increased the spreading of the virus in these areas in recent weeks. Thus, these parts of the country are more vulnerable to COVID-19, and a special attention needs to be paid in these parts of the country to control the further spreading.

Additionally, to get the most infected districts by COVID19 , we analyzed and ranked the top 15 districts based on the percentage distribution of cases, as shown in Table 6 . As of 08 August 2020, Dhaka was the most affected district and 


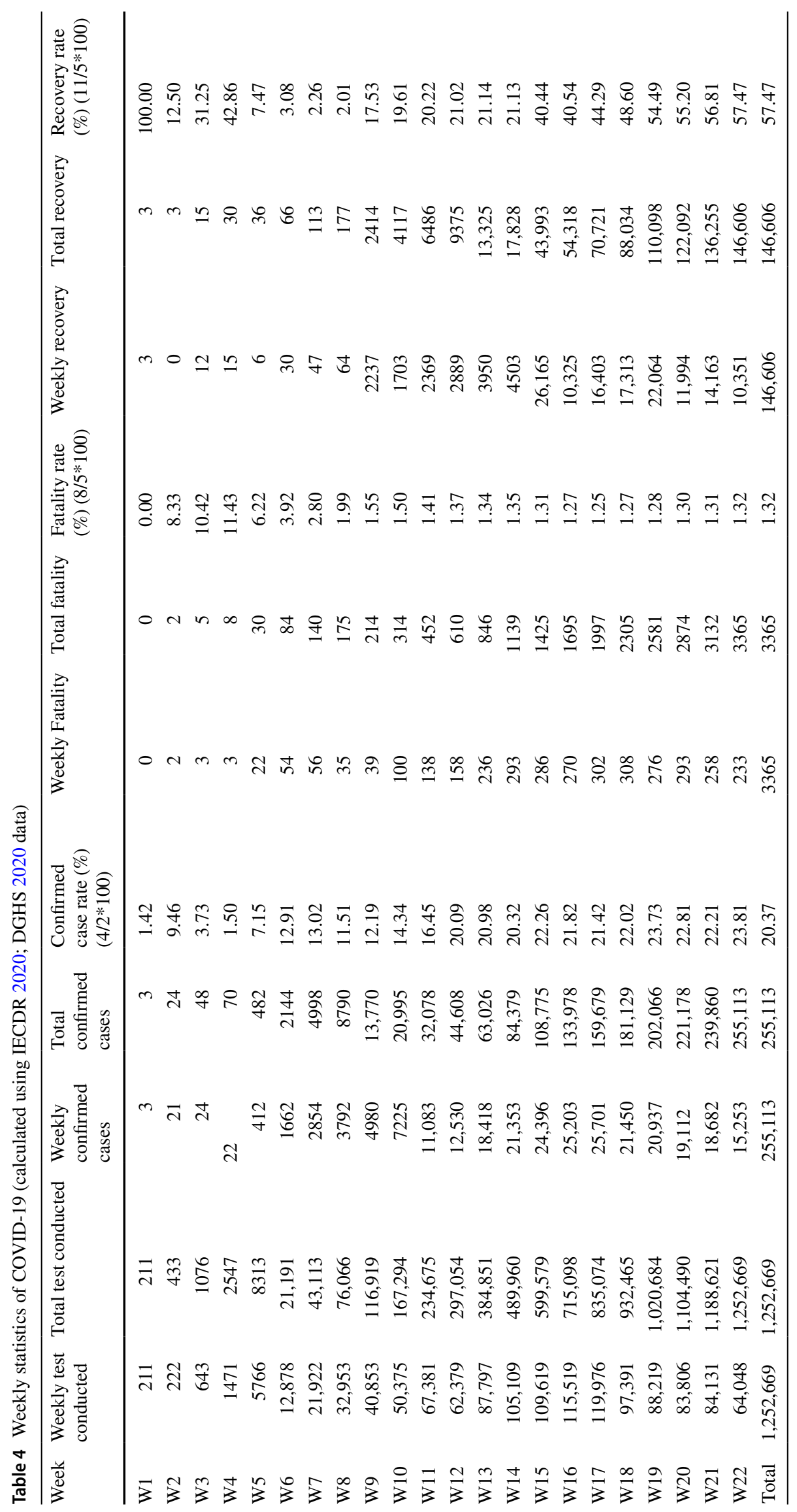


Table 5 Geo-temporal spreading of first confirmed cases over time by district

\begin{tabular}{|c|c|c|c|c|}
\hline Month & Day & District & Total & $\begin{array}{l}\text { Total } \\
\text { by } \\
\text { month }\end{array}$ \\
\hline \multirow[t]{5}{*}{ March 2020} & 08 & Madaripur, Narayanganj & 2 & 8 \\
\hline & 15 & Dhaka, Gazipur & 2 & \\
\hline & 19 & Chuadanga, Gaibandha & 2 & \\
\hline & 24 & Cox’s Bazar & 1 & \\
\hline & 27 & Chattogram & 1 & \\
\hline \multirow[t]{19}{*}{ April 2020} & 02 & Jamalpur, Shariatpur & 2 & 55 \\
\hline & 05 & Manikganj, Moulovi Bazar, Sherpur, Sylhet & 5 & \\
\hline & 06 & Kishoreganj & 1 & \\
\hline & 08 & Cumilla, Narshingdi, Nilphamari, Rajbari, Rangpur, Tangail & 6 & \\
\hline & 09 & Gopalganj, Munshigonj & 2 & \\
\hline & 12 & $\begin{array}{l}\text { Barguna, Hobiganj, Jhalokathi, Laksmipur, Netrokona, Lalmonirhat, } \\
\text { Chandpur, Mymensingh, Panchagar, B.Baria, Potuakhali, Thakur- } \\
\text { gaon }\end{array}$ & 12 & \\
\hline & 13 & Barishal, Rajshahi, Sunamganj & 3 & \\
\hline & 14 & Faridpur, Khulna, Kurigram, Narail, Pirojpur & 4 & \\
\hline & 15 & Dinajpur, Noakhali & 2 & \\
\hline & 17 & Bandarban, Bogra, Feni, Joypurhat, Pabna & 5 & \\
\hline & 18 & Bagerhat, Jessore & 2 & \\
\hline & 20 & Sirajganj & 1 & \\
\hline & 21 & Chapainawabganj, Naogaon & 2 & \\
\hline & 22 & Kushtia, Magura, Meherpur & 3 & \\
\hline & 23 & Natore & 1 & \\
\hline & 24 & Bhola & 1 & \\
\hline & 26 & Jhenaidah & 1 & \\
\hline & 29 & Satkhira & 1 & \\
\hline & 30 & Khagrachari & 1 & \\
\hline May 2020 & 07 & Rangamati & 1 & 1 \\
\hline Total & & & 64 & 64 \\
\hline
\end{tabular}

accounts for the highest $34.44 \%$ of the total cases. Chattogram, Narayanganj, Cumilla, and Bogra were the second, third, fourth, and fifth ranked districts, respectively. Remarkably, the top 15 districts account for about $73 \%$ of the total cases. Thus, these districts are more exposed districts judging by the percentage of total infection of COVID19 , and mainly located in the central and eastern parts except Rajshahi, Bogra, and Khulna (western part) [Fig. 12a]. The spatial pattern of the highest distribution of cases by districts signals that the number of victims was higher in the eastern part than in the western part. The eastern and western parts of Bangladesh are sharply divided through channels of the mighty rivers Jamuna and Padma (Fig. 12a). This acts as a physical barrier for the east-west connectivity and mobility, and, hence, it may influence in the spreading of the disease, COVID-19. However, details geographical research are required on this issue to get a clear picture.

The changing nature of the transmission of disease over time and space is crucial to understand the spatial dynamics of transmission. As seen in Fig. 13a, out of 14 top-ranked districts, the percentage distribution of the total cases reduced in three districts, namely Dhaka, Narayanganj, and Gazipur over time. Conversely, this proportion was increased in the remaining 11 districts, and particularly, from the week 14, the cases increased rapidly in these districts. The increase of the cases in several districts in later weeks proves that the disease has spread out across the country and intensity of the infection has also increased at a wider scale. Again, complementarily to Fig. 13a, we present in Fig. 13b further the temporal and spatial progression of the disease dynamics by calculating the correlation coefficient between the time (weeks 1-22) and the percentage of cases by districts. The figure signals that the districts where the disease outbreaks and concentrated initially; the number of victims has decreased in those districts over time (negative value of correlation coefficient, $r$ ). The districts having a positive coefficient $(r)$ represents an increase in the number of cases over time, and particularly, the high $r$ value indicates a high rate of increase of the cases and vice versa. Thus, the spatial dynamics of the reported cases indicates that the spreading 


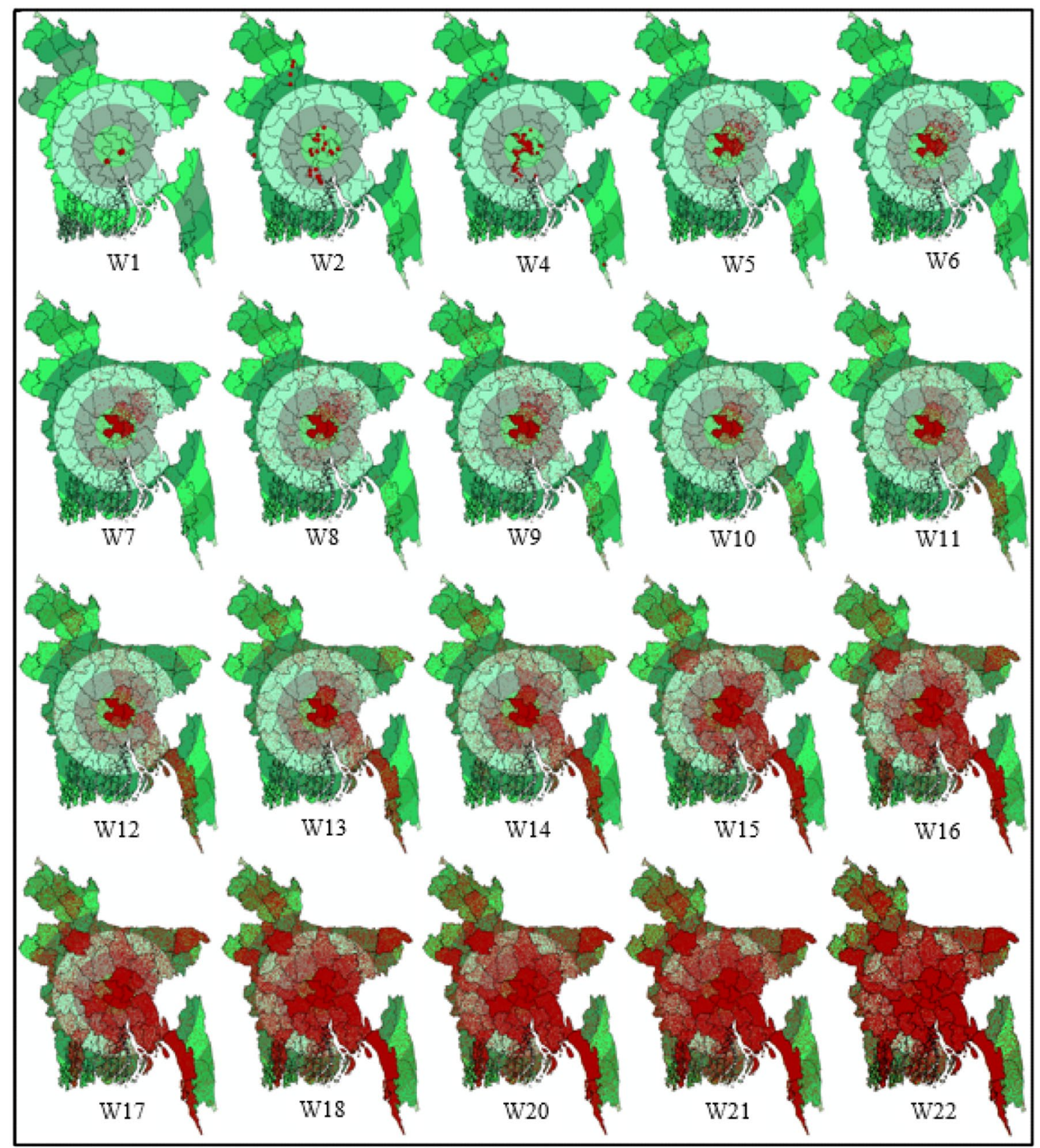

Fig. 11 Spatiotemporal distribution and pattern of the COVID-19 transmission in Bangladesh by districts and epidemiological weeks. Each circle in the background represents $50 \mathrm{~km}$ buffer zone from the centre point of Dhaka. $W$ week

of infection was taking place more in the western, southeastern, and north-eastern districts, and transmitted nationwide in later weeks (weeks 14-22) [Fig. 13b].

\section{Spatiotemporal pattern: reported COVID-19 deaths}

Deaths, due to the outbreak of any infectious disease, is a vital concern, since it acts as an important indicator of the severity of the disease. Results on the temporal and spatial distribution and pattern of the dynamics of death due to COVID-19 are presented in Fig. 14. Here, we symbolised the distribution of the number of deaths by district and epidemiological week (weeks 7-22). The figure illustrated that initially the death toll was limited and concentrated only to Dhaka and its adjoining district Narayanganj, and until week 11, the number of fatalities was high in this two districts and some surrounding districts within 50-100 km. However, in week 14, the death toll increased remarkably compared to the previous weeks, and with 293 deaths in this week, the total fatality crossed 1000 level (1139) [Table 4]. 
Table 6 The top 15 ranked districts based on the percentage distribution of the total cases and deaths

\begin{tabular}{|c|c|c|c|c|c|c|c|}
\hline \multicolumn{4}{|c|}{ Confirmed cases (as of 08 August 2020) } & \multicolumn{4}{|c|}{ Deaths (as of 08 August 2020) } \\
\hline Rank & District & $\%$ of total cases & Cumulative & Rank & District & $\%$ of total death & Cumulative \\
\hline 1 & Dhaka & 35.64 & 35.64 & 1 & Dhaka & 29.87 & 29.87 \\
\hline 2 & Chattogram & 7.88 & 43.52 & 2 & Chattogram & 7.79 & 37.66 \\
\hline 3 & Narayanganj & 3.19 & 46.71 & 3 & Cumilla & 5.56 & 43.22 \\
\hline 4 & Cumilla & 3.03 & 49.74 & 4 & Narayanganj & 4.40 & 47.62 \\
\hline 5 & Bogra & 2.74 & 52.48 & 5 & Bogra & 3.51 & 51.13 \\
\hline 6 & Faridpur & 2.73 & 55.21 & 6 & Sylhet & 3.33 & 54.46 \\
\hline 7 & Khulna & 2.46 & 57.67 & 7 & Chandpur & 2.64 & 57.10 \\
\hline 8 & Sylhet & 2.43 & 60.10 & 8 & Munshigonj & 2.38 & 59.48 \\
\hline 9 & Gazipur & 2.30 & 62.40 & 9 & Noakhali & 2.32 & 61.80 \\
\hline 10 & Rajshahi & 1.87 & 64.27 & 10 & Khulna & 2.20 & 64.00 \\
\hline 11 & Cox'sbazar & 1.87 & 66.14 & 11 & Mymensingh & 2.14 & 66.14 \\
\hline 12 & Noakhali & 1.81 & 67.94 & 12 & Cox's Bazar & 1.87 & 68.01 \\
\hline 13 & Munshigonj & 1.69 & 69.63 & 13 & Gazipur & 1.87 & 69.88 \\
\hline 14 & Mymensingh & 1.60 & 71.24 & 14 & Barishal & 1.55 & 71.43 \\
\hline 15 & Barishal & 1.37 & 72.60 & 15 & Faridpur & 1.55 & 72.98 \\
\hline 16 & Other districts & 27.40 & 100.00 & 16 & Other districts & 27.02 & 100.00 \\
\hline
\end{tabular}
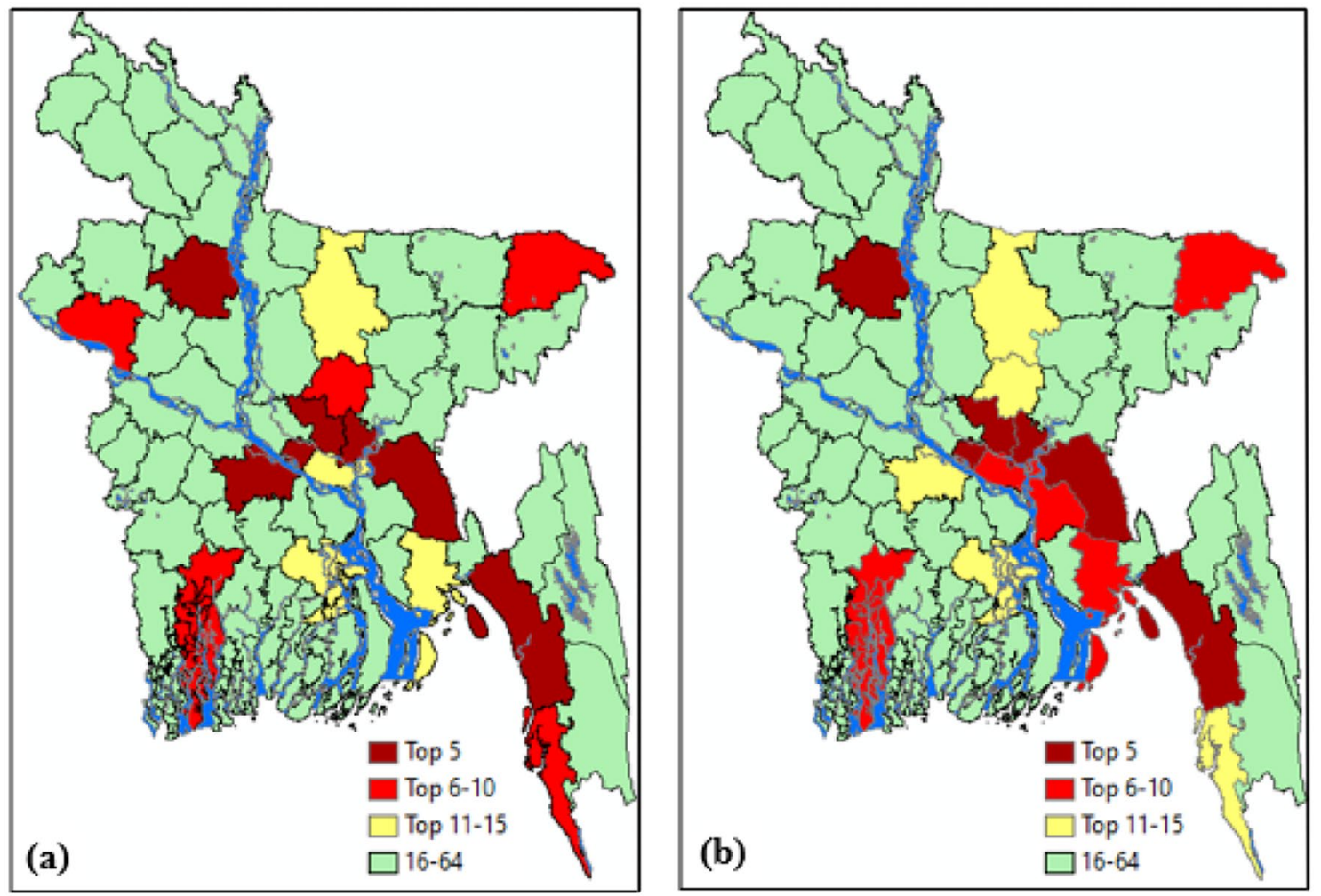

Fig. 12 The top 15 ranked districts based on the percentage distribution of the total numbers: a reported cases and $\mathbf{b}$ reported deaths [as of 08 August 2020]

Chattogram and Cox's Bazar, the two south-eastern districts, came up as second intensified areas of deaths in this week and later. Furthermore, Fig. 14 reports, in weeks 17-22, the death toll remarkably took place in the central, southeastern, north-eastern, and some north-western and southwestern districts. Thus, concerning deaths incidence, these 


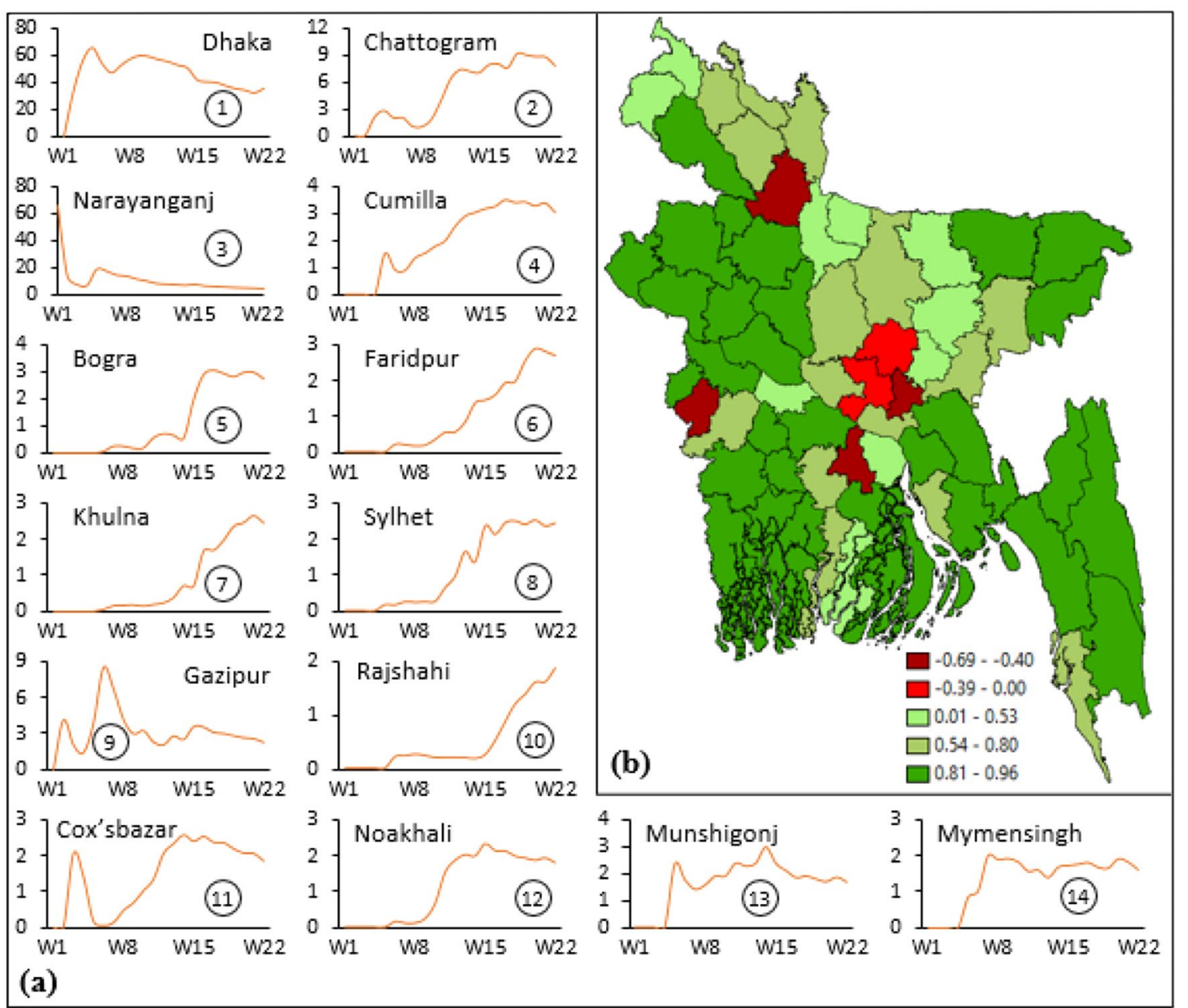

Fig. 13 a Temporal spreading: percentage distribution of cases in the top 14 districts, $x$-axis represents week and $y$-axis represents $\%$ of total cases; b correlation coefficient ( $r$ ) distribution between time (weeks 1-22) and percentage of the cases by districts

parts of the country are highly vulnerable to COVID-19. Again, the comparison between the spatial distribution of the deaths (Fig. 14) and the cases (Fig. 11) indicates that where the number of victims/infections was high, the number of fatalities was also high and vice versa. Therefore, it is necessary that with the improvement of the health system, measured should be taken to reduce the number of infections as well in the highly concentrated areas.

Similar to the cases distribution analysis, to get the most affected districts by deaths, we evaluated and ranked the top 15 districts based on the percentage distribution of deaths, as shown in Table 6. As of 08 August 2020, Dhaka was the most affected district and accounts for the highest $29.87 \%$ of the total deaths. Chattogram, Cumilla, Narayanganj, and Bogra were the second, third, fourth, and fifth affected ranked districts, respectively. The top 15 districts account for about $73 \%$ of the total deaths, meaning that these districts were the most exposed districts judging by the percentage of total deaths. These districts are mainly located in the central and south-eastern parts of the country except Bogra, Sylhet, and Khulna districts [Fig. 12b]. The spatial pattern of the highest distribution of deaths by districts shows that the number of deaths was higher in the eastern part compared to the western part of the country. As mentioned earlier that the eastern and western parts are divided by the rivers channel and may influence in the spreading of the disease, it may also influence death incidence. The spatial dynamics of fatality, as seen in Fig. 15a reports, out of 14 top-ranked districts, the percentage distribution of the total deaths reduced in three districts, namely Dhaka, Narayanganj, and Mymensing over time. The proportion was increased in the remaining 11 districts, especially from the week 14 (Fig. 15a). The increase in the number of deaths in several districts in week-14 and later evidences that the occurrence and intensity of the death amplified across the country at a wider scale. Complementarily to Fig. 15a, we present in Fig. $15 \mathrm{~b}$ further the temporal and spatial progression of the dearth dynamics by calculating the correlation coefficient 


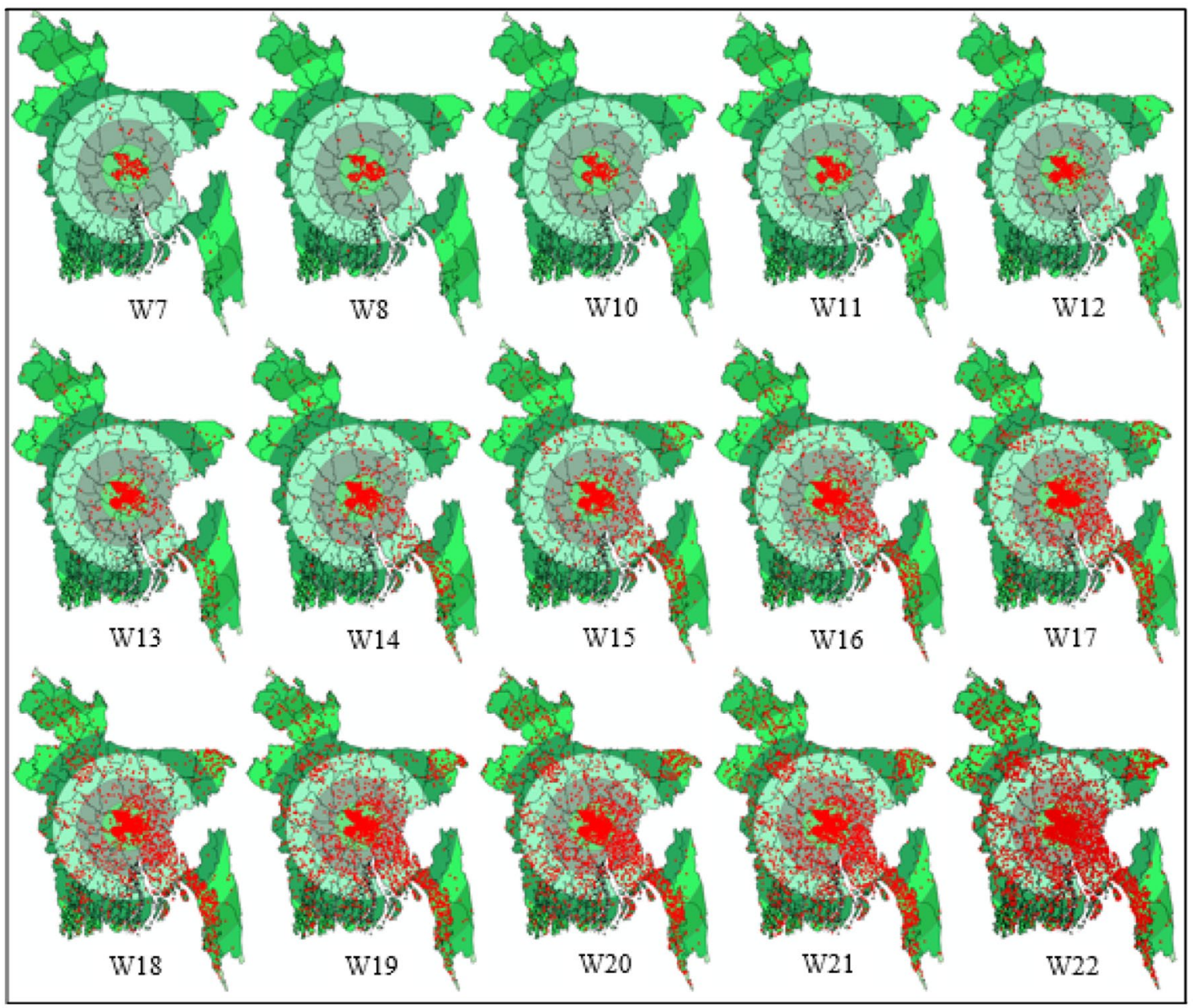

Fig. 14 Spatio-temporal distribution and pattern of the COVID-19 deaths in Bangladesh by districts and epidemiological weeks (weeks 7-22). Each circle in the background represents $50 \mathrm{~km}$ buffer zone from the centre point of Dhaka. $W$ week

between the time (weeks 7-22) and the percentage of deaths by districts. The figure points out that the districts where the deaths initially reported and concentrated more, the number of deaths decreased in almost all of those districts over time (negative value of correlation coefficient, $r$ ). The districts having a positive coefficient $(r)$ represents an increase in the number of deaths over time, and particularly, the high $r$ value indicates a high rate of increase of the deaths and vice versa. Thus, the spatial dynamics of the reported deaths, as seen in Fig. 15b, which indicates that the number of deaths increased more in the south-western and north-western districts in later weeks (weeks 14-22) [Fig. 15b].

\section{Spatiotemporal hot spots (reported cases and deaths)}

Hotspot analysis is a spatial analysis and mapping technique interested in the identification of clustering of spatial phenomena. Space-time pattern mining allows for the detection of statistically significant hot and cold spots within an area. It also allows to identify trends and defines whether the hot or cold spot is persistent, increasing, or decreasing. To detect the statistically significant hot and cold spots of the incidence of the infected cases and deaths, here, we used Getis-Ord Gi* (G-i-star) statistic (Getis and Ord 1992) and $Z$ score, $p$ value, and classification of each bin were calculated and mapped. The resultant $z$ scores and $p$ values tell where features with either high or low values cluster spatially. Thus, the output of the G-i-star analysis demonstrated the locations of hot and cold spots, as highlighted in Fig. 16. The spatio-temporal analysis of three selected times hotspots shows a high spatial clustering hotspot in the central part of the country for both the cases and death distribution. This core locations of hotspots include Dhaka, Narayanganj, Gazipur, Narshingdi, Munshiganj, and Manikganj districts were the most affected areas in terms of infection and related deaths (Fig. 16). After time being, in week 22, a second high spatial clustering hot spot was identified in the north-eastern 


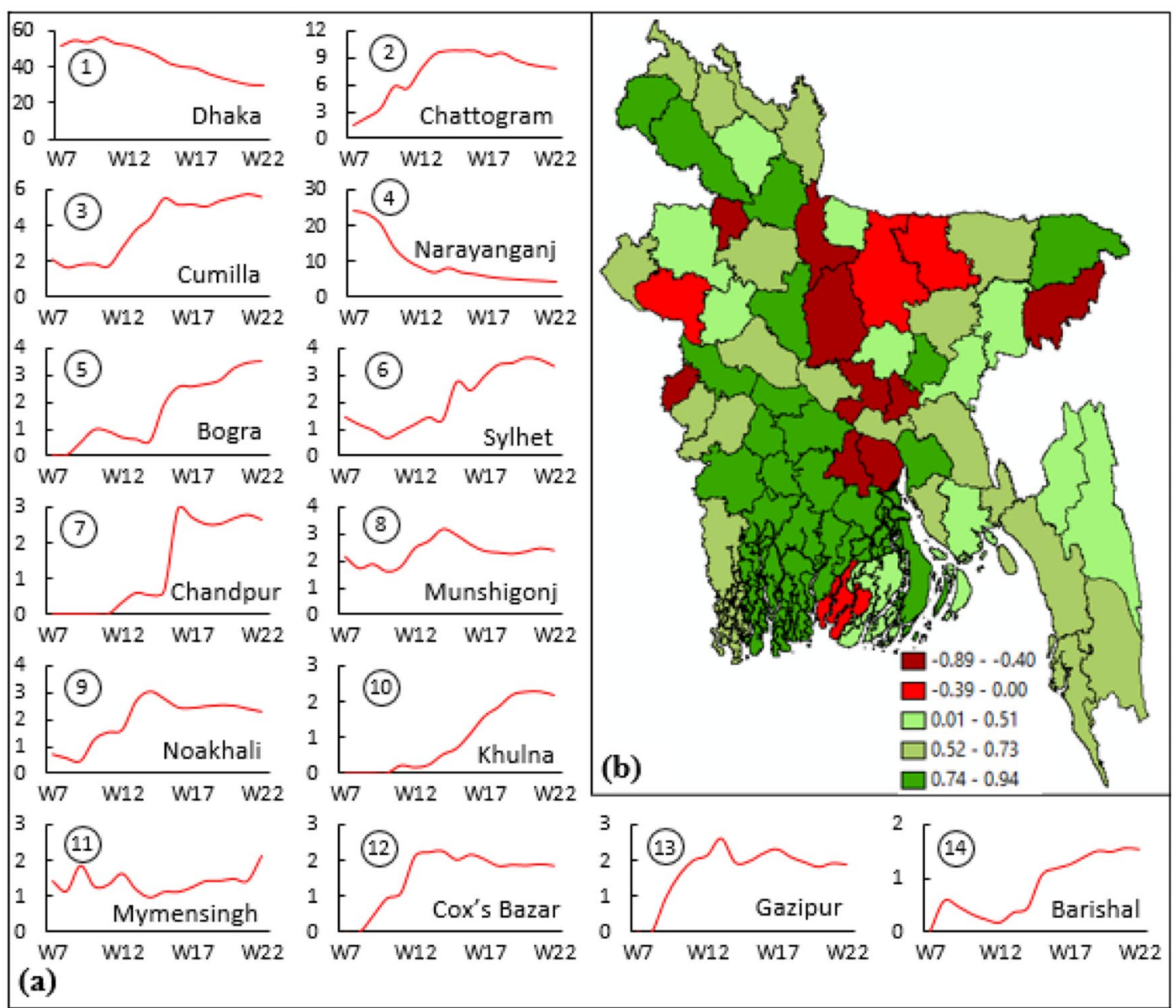

Fig. 15 a Temporal spreading: percentage distribution of deaths in top 14 districts, $x$-axis represents week and $y$-axis represents $\%$ of the total deaths; $\mathbf{b}$ spatial distribution of the correlation coefficient $(r)$ between time (weeks 7-22) and percentage of deaths by districts

district namely Cartogram, exhibiting that the epidemic situation in this area was deteriorated and the incidence rate was increased. Again, comparatively low spatial clustering but emerging separate hotspots (isolated clustering pattern) were also recognized in Sylhet, the north-eastern district and Bogra, the north-western district in week 22 for both the reported cases and deaths, indicating that there is a possibility of developing more single clustering patterns in some other parts of the country if the situation is not controlled. The results of the hotspots analysis pointed out that the outbreak presented a strong clustering pattern during the period of study, and the spatiotemporal clustering pattern of COVID-19 was dominated mainly by a single clustering pattern and concentrated significantly in the central part, and spread out mainly from the central (capital and neighbouring districts) to the south-eastern part of the country. High population density, high urban population and housing density, location of the economic hubs, migrant households, concentration of industry/ factory workers, etc. gear up the spreading of the virus in these areas and make these areas as hotspots to the disease.

\section{COVID-19: vulnerability zoning (Cov19VZ)}

The outbreak of COVID-19 is an unexpected threat to inhabitant worldwide. Since there is no effective medication against COVID-19 virus, management practices to minimize this problem can be effectively carried out if the magnitude, spatial distribution and transmission pattern, and vulnerability of COVID-19 are known. Since this study was able to identify the spatial pattern of the transmission and dynamics of the disease along with hotspots, the spreading and the magnitude of the disease in a particular location/ area somehow could be related to many associated inherent conditions and environmental setups of the area. It is clear that COVID-19 virus transmitted from human to human; therefore, the transmission of virus depends on human nature and behaviour, mobility, and social and economic 

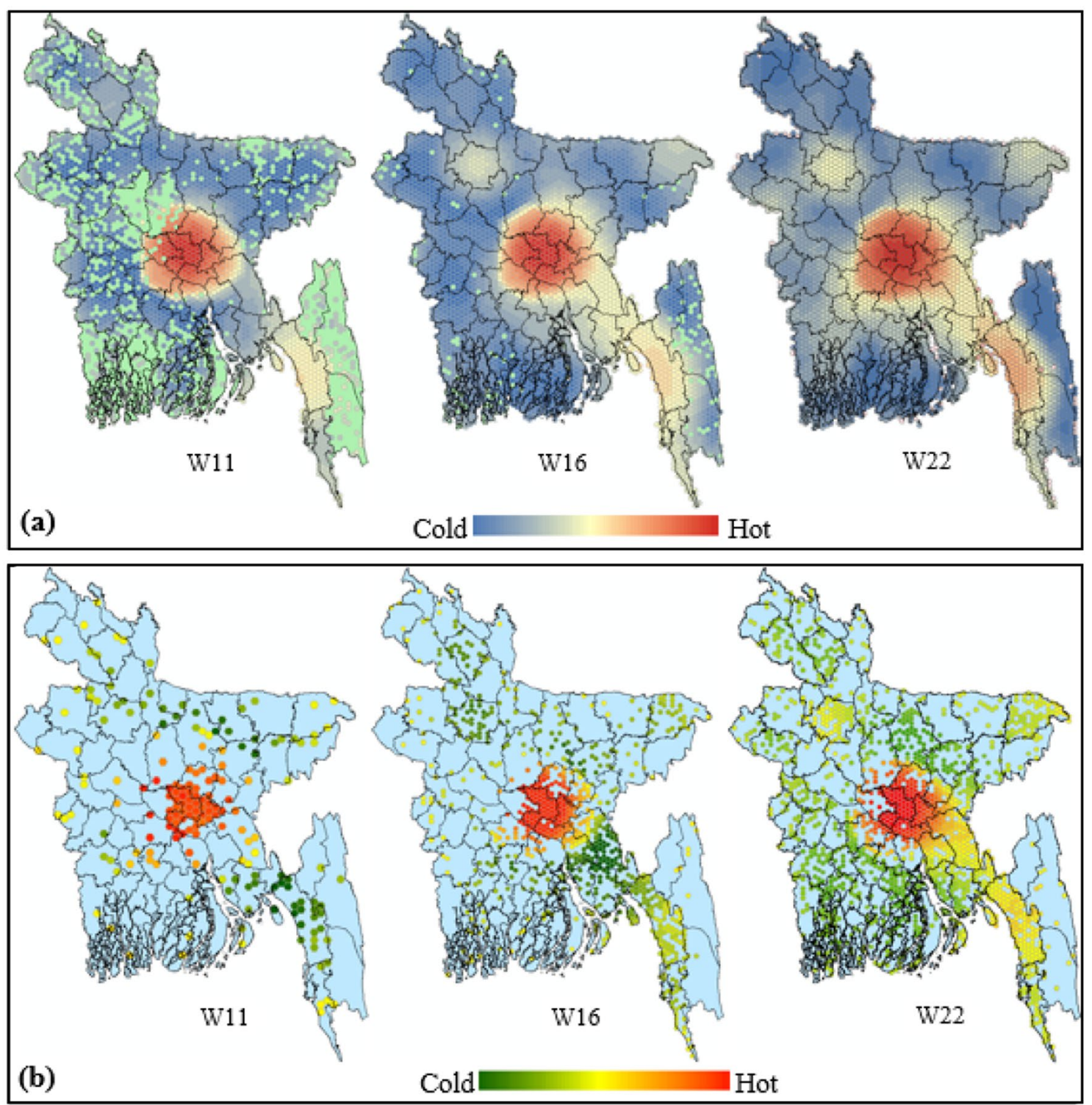

Fig. 16 COVID-19 spatio-temporal hotspots: a reported cases and $\mathbf{b}$ deaths

conditions/status of the inhabitants of the area. Thus, for effective management, COVID-19 vulnerability zoning (Cov19VZ) based on some bio-socio-economic factors can be an essential tool in an outbreak area, as vulnerability zoning explains and displays the distribution of areas that likely to be affected to different magnitudes. Viewing this, based on the spatial pattern and dynamics of the cases and deaths distributions, combined professional expert judgment and relationship between the cases distribution and factors; the dominating twelve (12) factors were selected and assessed for the Cov19VZ. The selected relevant 12 factors were (i) total industry/factory worker, (ii) total establishments employee, (iii) monthly household consumption, (iv) floating population, (v) male population, (vi) number of migrants, (vii) population density, (viii) urban male population, (ix) urban population, (x) senior citizen (above 60 age group), (xi) working-age population (21-59 age group), and (xii) literacy rate (Fig. 17). Urban areas tend to have more cases and deaths compared to rural areas, the infection more often occurs in crowded places, workers and employees need to move frequently to their workplaces to earn and meet the consumption of the family, the transmission of the virus and related deaths are often associated with the age group and gender issue, and migrants and their family members incline to have more possibilities to spread of the disease, etc. were under the consideration to select factors primarily. Before selecting these factors, finally, we also analyze the relationship between factors and the distribution of the cases 


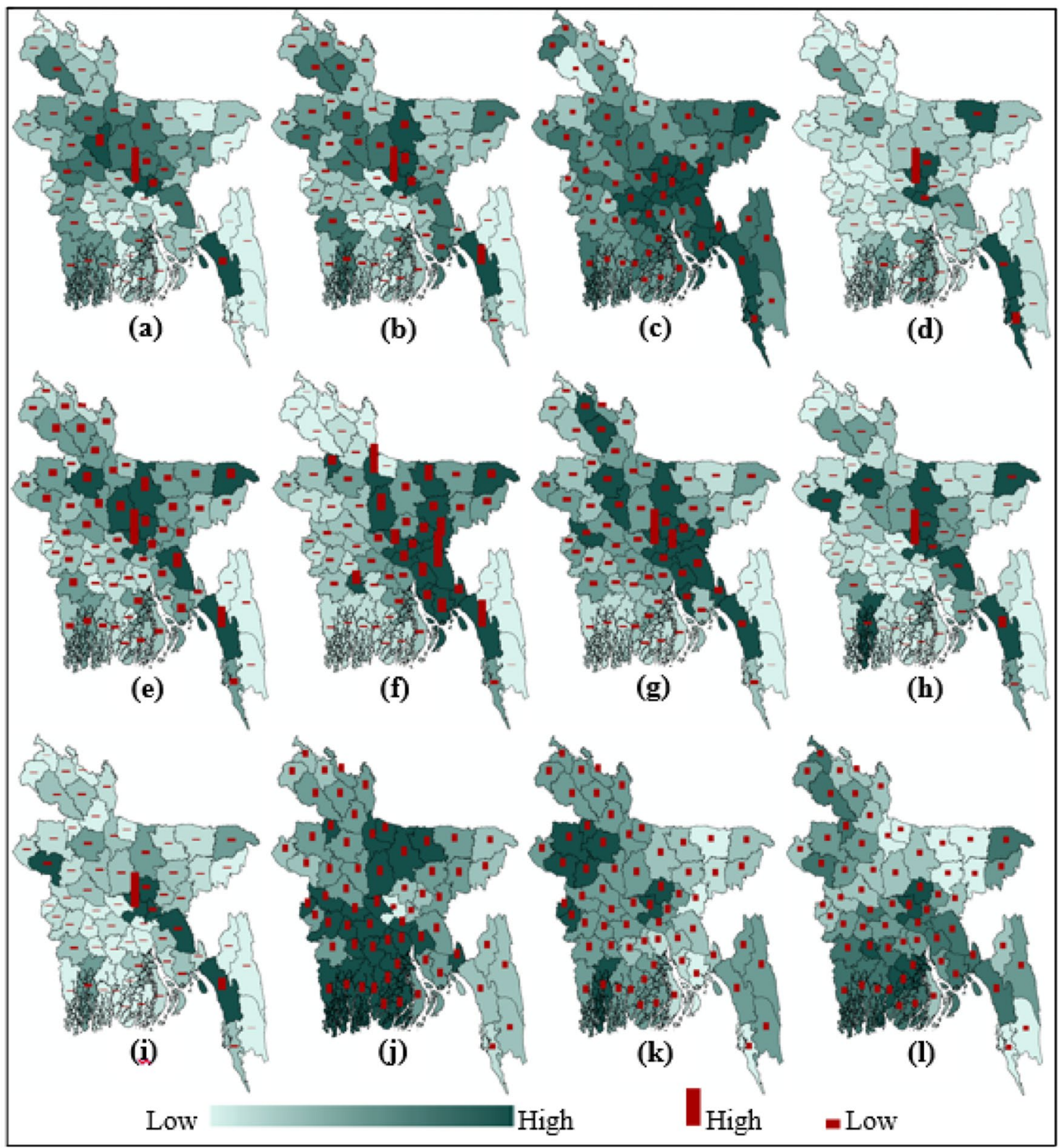

Fig. 17 Selected factors for COVID-19 vulnerability zoning: a total industry/factory worker b total establishments employee, $\mathbf{c}$ monthly household consumption, $\mathbf{d}$ floating population, $\mathbf{e}$ male population, $\mathbf{f}$ number of migrants, $\mathbf{g}$ population density, $\mathbf{h}$ urban male population, $\mathbf{i}$ urban population, $\mathbf{j}$ senior citizen [above 60 age group], $\mathbf{k}$ workingage population [21-59 age group]), and $\mathbf{l}$ literacy rate of COVID-19 in different times (here weeks) by districts through correlation coefficient $(r)$, to know how strongly the factors are related to the distribution of the reported cases. As seen in Fig. 18, initially, the number of cases was very few that's why the $r$ value was low for all the factors; however, as the number of cases increases, the $r$ value also increases, representing strong relationships between the cases distribution and the factors except literacy rate. The comparatively low relationship in literacy rate indicates that both literate and illiterate people either were not aware or ignoring/violating the intervention measures which have been taken to reduce the transmission of the virus. However, for the urban population, urban male population, total establishments employee, and male population, we got very high $r$ values followed by the number of migrants, monthly household consumption, and total industry/factory worker by districts. $r$ value of population density rightly represents that spreading of the cases initially was higher in the high densely populated areas and later spread gradually to other parts.

In this study, in another way, we consider to find out the interactive effects of selected factors for COVID-19 
Fig. 18 Temporal distribution of correlation coefficient $(r)$ between selected factors and reported cases by district. $X$-axis and $y$-axis represent time (weeks) and correlation coefficient $(r)$, respectively

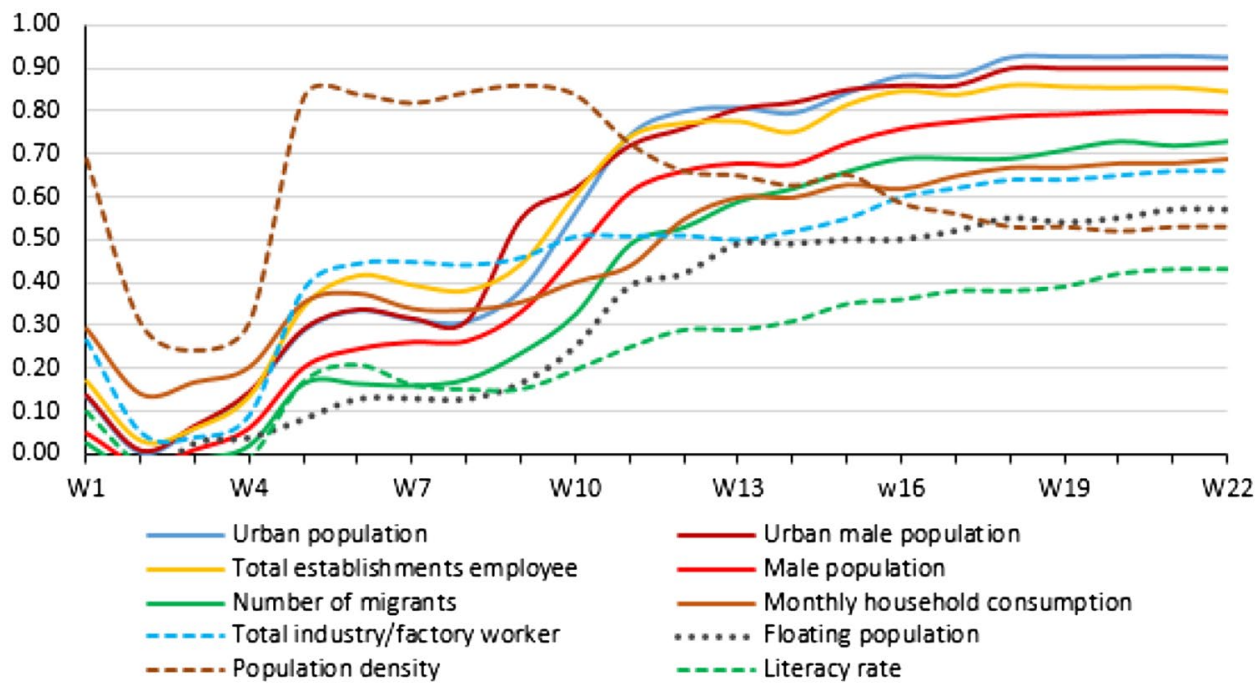

vulnerability zoning. Using GIS-aided AHP-WSM method, above-mentioned factors, and calculated COVID-19 vulnerability zoning index (Cov19VZI), we developed a districtlevel Cov19VZ map, as shown in Fig. 19a. The figure represents the magnitude of the vulnerability (as high, medium and low) of each district of Bangladesh. The spatial pattern of the vulnerability zoning of COVID-19 illustrated that the high vulnerable zone was mainly concentrated in the central, eastern, and south-eastern parts as a compact zone. Also, two districts in the south-western, one in the central-southern, one in the north-eastern, and two more in the north-western parts belong to this zone separately, indicating the most vulnerable area of the country to COVID19 threat (Fig. 19a). The medium level of vulnerable areas was widely distributed in the western, south-western, northwestern, and north-eastern parts of the country. On the

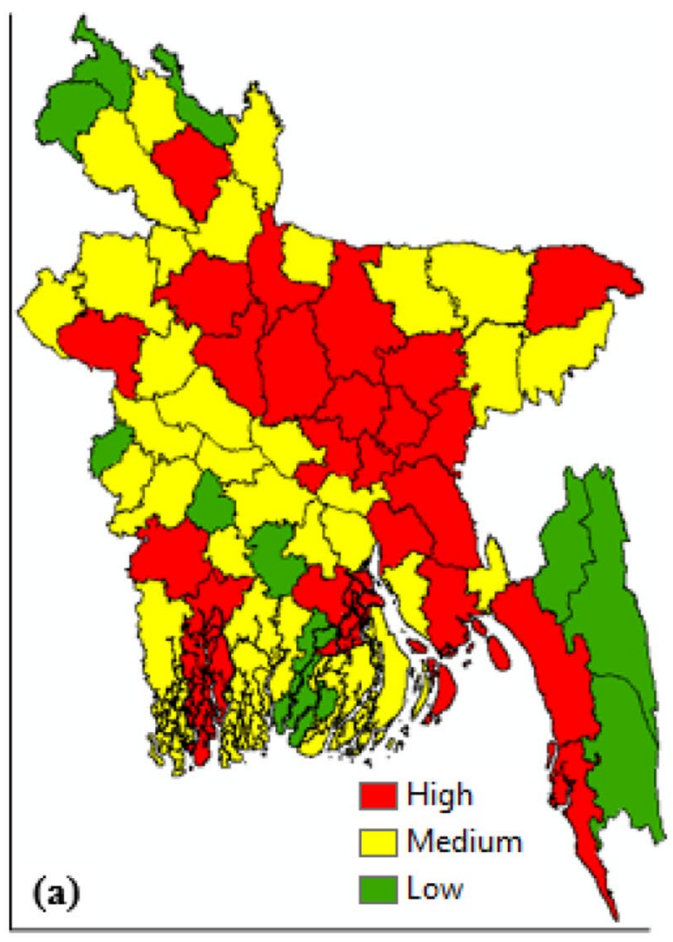

Fig. 19 COVID-19 vulnerability zoning (Cov19VZ) and model validation: a vulnerability zoning; b relationship between Cov19VZI and reported cases; c relationship between Cov19VZI and deaths. $X$-axis

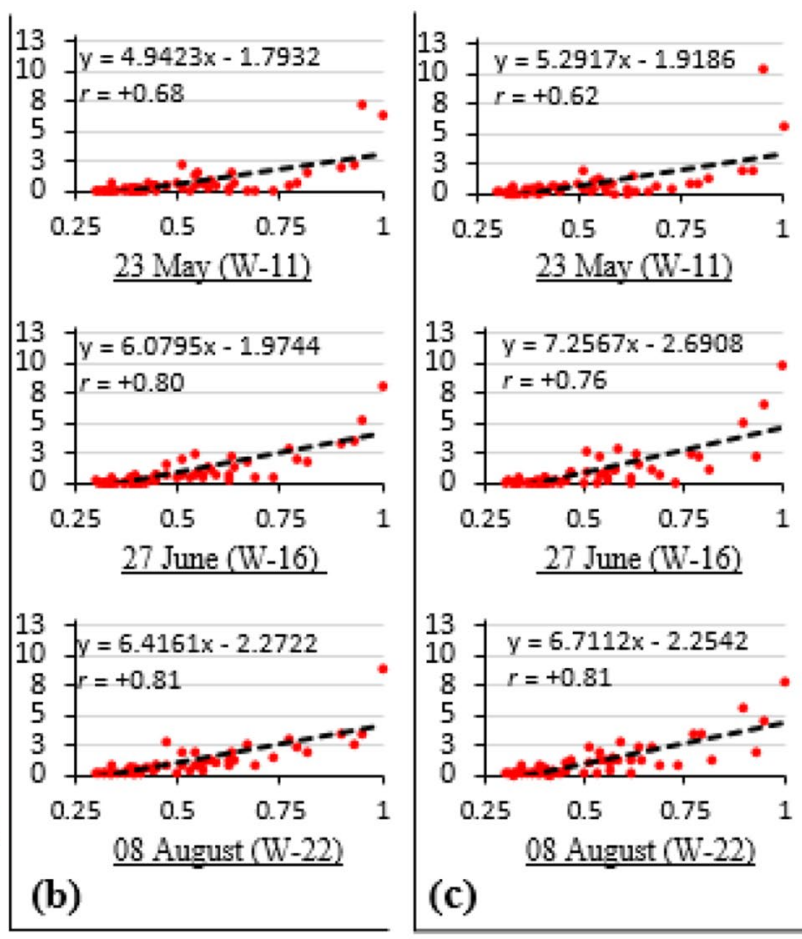

and $y$-axis represent Cov19VZ index and percentage distribution of cases/deaths, respectively 
contrary, the area under low vulnerability was mainly found in the south-eastern and extreme north-western parts, and scattered way in a few districts in the south-western region (Fig. 19a). Again, Table 7 further depicts that out of 64 districts, 22, 31, and 10 districts were found to be under high, medium, and low vulnerable areas, respectively. The Dhaka and Chattogram were the most vulnerable divisions having the highest number of districts under high vulnerable zone (six districts in each division), followed by Rajshahi (3), and Khulna (2) divisions. (Table 7). Thus, the vulnerability zoning of COVID-19 exposed that the degree of vulnerability has a geographical horizontal belt distribution, and in general, the vulnerability was comparatively high in the eastern part compared to the western part of the county. Size of the urban population, the male population including urban male, number of employees in establishments and workers in industry/factory, number of migrants, and, of course, population density of the district influence the spreading of the disease and make the area more vulnerable. Therefore, it is recommended that proper attention needs to be paid in high and medium vulnerable zones on a priority basis in controlling the spread of the disease.

To validate the effectiveness of the predictive vulnerable area zone map of COVID-19 (Fig. 19a), the vulnerability zoning index (VAZI) was spatially correlated with the case and death distribution by districts for three different times, Week 11, Week 16, and Week 22. Here, the coefficient of correlation $(r)$ was computed using the IDRISI TerrSet GIS and image processing software, and plotted with trend line (regression) [Figs. 19b and 18c]. Linear correlation coefficient analysis between Cov19VZI and reported cases revealed that these two variables were positively correlated at all the three weeks $(r=+0.68,+0.80$, and +0.81 at week 11 , week 16 , and week 22 , respectively), and the value of $r$ increased with time (Fig. 19b). On the other hand, the correlation coefficient between Cov19VZI and reported deaths showed that the distribution of the deaths was positively correlated with the vulnerable area at all the three times $(r=+0.62,+0.76$, and +0.81 at week 11 , week 16 , and week 22, respectively), and the value of $r$ also increased with time (Fig. 19c). Increasing of $r$ value over time also highlighted the dependency of the occurrences on the degree of vulnerability (graded vulnerable zone). Thus, correlation coefficient analysis ascertains that the number of cases and deaths was associated with the degree of vulnerability, and both the cases and deaths were increased with respect to the increased of VAZI, which validated the vulnerability zoning distribution described here. Hence, the vulnerability zoning can be used as a basic unit area for the management planning of COVID-19 situation, and it can also be very useful to planners and policymakers initiating remedial measures based on prioritizing areas.

\section{COVID-19 management strategies}

It was mentioned earlier that there is no effective medication against COVID-19 virus yet, and therefore, management practices to reduce the propagation and related deaths can be effectively carried out if the magnitude, spatial distribution and transmission pattern, and vulnerable areas to COVID19 information are available. One of the objectives of our study was to provide a comprehensive management strategy to cope with and control the spreading of the disease. The developed vulnerability zoning map of COVID-19 can be incorporated into management strategies and planning decisions. For formulation the effective management policies, the vulnerability zoning can be used as basic unit areas, and this may help to formulate a comprehensive management plan for each zone with careful consideration of certain activates. Worldwide accepted practices of NPIs are lockdown, isolation, quarantine, maintaining social distance, using protecting kits (face mask, hand sanitizer, hand gloves), etc. However, lockdown and complete isolation are more effective in the early days of the outbreak of the disease, if it is implemented effectively (Ferguson et al. 2020; Chan et al. 2020; Masrur et al. 2020; Chinazzi et al. 2020). Nevertheless, these cannot be effectively implemented for a long time, since it has an impact on socio-economic settings, particularly, in a developing country, like Bangladesh. Similar findings were found in our study too. Therefore, from these perspectives, a comprehensive management strategies need to be formulated. Table 8 presents an example of overall management strategies based on spatial dynamics, vulnerable area zoning, expert opinion, and guideline provided by the WHO and DGHS. These strategies could serve as basic

Table 7 Number of districts by division and vulnerability zoning

\begin{tabular}{lllllllll}
\hline Vulnerability Zone & \multicolumn{2}{l}{ Division } & \multicolumn{2}{c}{ Total } \\
\cline { 2 - 8 } & Dhaka & Chattogram & Rajshahi & Khulna & Sylhet & Barishal & Mymensing & Rangpur \\
\hline High & 6 & 6 & 3 & 2 & 1 & 1 & 2 & 1 \\
Medium & 6 & 2 & 5 & 6 & 3 & 3 & 2 & 4 \\
Low & 1 & 3 & - & 2 & - & 2 & - & 31 \\
Total & 13 & 11 & 8 & 10 & 4 & 6 & 4 & 8 \\
\hline
\end{tabular}


Table 8 Overall management strategies for COVID-19 based on vulnerability zoning-Bangladesh

\begin{tabular}{|c|c|c|c|}
\hline $\begin{array}{l}\text { Vulnerability } \\
\text { zoning }\end{array}$ & $\begin{array}{l}\text { Reported cases } \\
\text { and deaths }\end{array}$ & $\begin{array}{l}\text { Planning } \\
\text { actions }\end{array}$ & Overall management strategies \\
\hline High & Medium-to-high & $\begin{array}{l}\text { Emergency response, } \\
\text { recovery and action }\end{array}$ & $\begin{array}{l}\text { Priority to be given on emergency basis to limit the movement of the people by } \\
\text { travel restrictions, partial lockdown can be implemented in some areas } \\
\text { High restriction should be implemented on daily life activities } \\
\text { Activities with mass gathering (socio-cultural) need to be closed for specific } \\
\text { time period } \\
\text { Educational institutes should be closed. If problem persist for longer, alternative } \\
\text { way may be applied, like net based virtual class and office works } \\
\text { Use of protective kits must be mandatory during outside movement, if possible } \\
\text { provide kits at free of cost or a subsidized rate } \\
\text { Involve the law and order enforcement authority to monitor the NPIs practices } \\
\text { Need to create/provide/expand necessary facilities to meet local daily demands } \\
\text { maintaining social distance } \\
\text { Initiative should be taken to share the information and awareness development } \\
\text { to the people of this area. Health facilities including testing facilities need to } \\
\text { be increased } \\
\text { To take steps to provide financial assistance to the poor } \\
\text { Emergency activities and works should be done in a limited extent and main- } \\
\text { taining with proper hygiene and health rules }\end{array}$ \\
\hline Medium & Low-to-medium & $\begin{array}{l}\text { Protection, response and } \\
\text { recovery }\end{array}$ & $\begin{array}{l}\text { High probability to increase the infection rate in these areas, therefore, need to } \\
\text { carry out a scheme for effective NPIs practices based on expert opinion and } \\
\text { research findings } \\
\text { Strictly need to be maintained NPI practices like social distancing, using protec- } \\
\text { tive kits, avoiding mass gathering, and social and cultural agglomeration } \\
\text { Use of face mask mandatory during outdoor activities } \\
\text { Involve the law and order enforcement authorities to monitor the NPI practices } \\
\text { Strictly implications of law to verify COVID-19 do's or don'ts } \\
\text { Community-based COVID-19 management program including sharing informa- } \\
\text { tion and awareness development activities should be introduced } \\
\text { Testing facilities need to be simplified and increased }\end{array}$ \\
\hline Low & Low-to-medium & $\begin{array}{l}\text { Preparedness and pro- } \\
\text { tection }\end{array}$ & $\begin{array}{l}\text { Need to be protected from further spreading and all of those activities that play } \\
\text { role in the spreading of infection should be controlled and monitored to keep } \\
\text { the zone safe } \\
\text { Mandatory to use a face mask outside } \\
\text { Community based COVID-19 management program including sharing informa- } \\
\text { tion and awareness development activities need to be introduced } \\
\text { Testing facilities need to be simplified and increased }\end{array}$ \\
\hline
\end{tabular}

components in a comprehensive COVID-19 management plan for the study area.

Since COVID-19 transmitted from human to human, the accelerated propagation of the disease mainly arises due to the activities, movement, behavioural pattern, carelessness, and unconsciousness of the people. It may also arise due to the lack of proper knowledge, management policies, and practices. Therefore, top priority should be given on taking the right steps and raising awareness of the people, proper planning, and appropriate management practices when formulating policy for the management of the COVID-19 circumstances. It may be mentioned here that the vulnerability zoning, i.e., high, medium, and low, can be treated as so-called red, yellow, and green zones, respectively, in the formulation management strategy and policy. The high vulnerable areas are the most significant areas to concentrate management effort due to their vulnerability. These areas are mainly distributed in the areas where the concentration of establishment employees, industry workers, population density, and the urban population including urban male and migrants are high. In the high vulnerable areas, the observed cases and deaths due to COVID-19 were recorded as medium-to-high, and these areas are the core areas of economic development. Moreover, most of the districts under this zone are within the hotspots cluster area of COVID19. Therefore, in these areas, priority should be given to reduce the rate of infection and recovery of the patients through emergency response, recovery, and action planning (Table 8). The medium vulnerable areas can be considered as focal regions for protection and recovery. Thus, the first 
step should be taken to protect the area from further infection, and then, the priority should be given to reduce the infection and recovery of the patients. The low vulnerable areas should be the key regions for strict protection due to reasonable rates of infection and associated deaths. This region was mainly located in the hilly areas and extreme north-western parts of the country, it will be difficult for the protection and recovery if the people infected more, since these areas are far away from the central business districts (Dhaka, Chattogram, and Rajshahi). Therefore, these areas need to be protected from further spreading, and all of those activities that play a role in the spreading of infection should be controlled and monitored to keep the zone safe.

\section{Conclusions}

This study was an effort to in-depth assess and analyze the COVID-19 outbreak and transmission dynamics through space and time in Bangladesh using 154 days (08 March-08 August 2020) real-time data series of confirmed cases and associated deaths. For geospatial analysis and modelling of the epidemic dynamics, hotspot identification, and vulnerability zoning (Cov19VZ), we conducted this study at the district level and analyzed using GIS. Overall, the study reveals that in Bangladesh, the status of the pandemic was in exposure level during the study period. And, though the Government has taken some effective measures to mitigate and control the spreading of COVID-19, lack of awareness, reluctance to follow the rules and regulations, ignoring to maintain the social distance, health safety measures, etc. increased the spreading of virus and worsen the situation in the recent weeks. The major findings of the temporal analysis were (i) in a large populated country, the number of test conducted per day was not enough, and as the number of tests increased, the number of positive cases increased and vice versa, thus, increasing the number of tests can be an effective measure to get the real picture; (ii) decreasing of the number of cases in recent time (particularly in weeks 18-22) does not reflect the recession period of infection, since the rate of confirmed cases (daily, weekly, and total) shows increasing/same trend; (iii) the disease transmitted at almost the same rate (between 20 and 24\%) from the last 12 weeks that considered as a high rate of infection; (iv) as of 08 August 2020, the cases were doubled in 11 days, denoting faster spreading; (v) the male people were infected more than the female $(71 \%$ verses $29 \%$ ), and younger were more infected compare to elder; (vi) the fatality rate was remained almost the same (1.1-1.3\%) for the last 12 weeks, and fatality rate was higher among male and elder people than female and younger (male $79 \%$ and female $21 \%$ ); and (viii) the recovery rate was nearly the same as the global average rate of recovery and as of 29 August 2020, recovery rate was about $62 \%$ of the total cases.
On the contrary, geo-spatial analysis exhibits that the disease propagates from the central parts of the country initially and then spread out in other parts over time. In the central, south-eastern, north-eastern, and some northwestern and south-western districts, the disease transmitted widely and at a high rate, and consequently, the death toll was also high in these districts. Dhaka was the most exposed district followed by Chattogram, Narayanganj, Cumilla, and Bogra (the top five ranked districts) based on the number of cases and deaths. A single strong clustering pattern in the central part, which spread out mainly to the south-eastern part, identified as a prime hotspot in both the cases of infection and deaths. Isolated low spatial clustering but emerging hotspots were also recognized in the north-eastern and north-western parts for both the reported cases and deaths, indicating the possibility of developing more single clustering patterns as hotspots in some other parts, if the situation is not controlled. This study was able to identify the potential linkages between the transmission of diseases and the selected bio-socioeconomic factors that gear up the spreading of the disease in the area. Based on selected bio-socio-economic factors and the method described, the COVID-19 vulnerability zoning categorized the central, eastern, and south-eastern parts as high vulnerable zone, and on the contrary, the western, south-western, north-western, and north-eastern parts as the medium vulnerable zone. The rest of the area was grouped as the low vulnerable zone. The Cov $19 \mathrm{VZ}$ exposed that the degree of vulnerability has a geographical horizontal belt distribution, and in general, the vulnerability was comparatively high in the eastern part compared to the western part, which consistent with the delineated top 15 ranked districts based on the percentage distribution of the reported cases and related deaths.

Finally, it can be concluded that, despite the space-timebased analysis of the transmission dynamics of COVID-19, we described a method for the modelling of vulnerability zoning supported by GIS-aided AHP-WSM and selected bio-socio-economic factors, and the method delineated vulnerable area quantitatively over a comparatively large area. The advantage of the method is the incorporation of spatially variables into the decision-making process to determine the degree of vulnerability. Thus, this could be useful as an analytical and decision-making tool for the incorporation of spatially variable risk perception in GIS-based decision support systems. Furthermore, the vulnerability zoning was validated against the distribution of the cases of infection and deaths with correlation coefficient and trend line (regression). The vulnerable zoning exercise in this study made it possible to identify the vulnerable areas with different magnitude that require urgent intervention through proper management and action plan (preparedness, emergency response, recovery, and action). Therefore, based on this study, comprehensive 
management strategies were anticipated (Table 8). As this study was involved in assessing, monitoring, and vulnerability zoning of the transmission of COVID-19 based on space and time, and provides a comprehensive management strategies, it is expected that this will be a useful guide towards understanding the space-time-based characteristics of the COVID-19 and assist to policy makers to prioritize resource allocation, and to formulate an effective management and action plan to mitigate the COVID-19 problem in Bangladesh. By appropriate adjustment of some factors with local relevance, COVID-19 vulnerability zoning index (i.e., Cov19VZI) derived by GIS-based AHP-WSM modelling along with relevant factors can be applied to other regions, and or any other infectious disease, in general. This method was applied at a regional scale, but if the data of determining factors are available at a larger scale, it could be applied to evaluate the vulnerability for small areas too. However, only some selected factors were considered; therefore, further application and validation of this method to more complicated landscapes may be needed.

Acknowledgements Authors are thankful to the anonymous reviewers for their valuable comments and suggestions.

Funding There is no funding issue as well.

\section{Compliance with ethical standards}

Conflict of interest There is no conflict of interest between the authors, and findings of the research.

\section{References}

Acharya R, Porwal A (2020) A vulnerability index for the management of and response to the COVID-19 epidemic in India: an ecological study. Lancet Global Health 8(9):E1142-E1151. https://doi. org/10.1016/S2214-109X(20)30300-4

Ainslie KEC, Walters C, Fu H et al (2020) Evidence of initial success for China exiting COVID-19 social distancing policy after achieving containment. WHO Collaborating Centre for Infectious Disease Modelling MRC Centre for Global Infectious, London

Aleta A, Moreno Y (2020) Evaluation of the potential incidence of COVID-19 and effectiveness of containment measures in Spain: a data-driven approach. BMC Medicine 18:157. https://doi. org/10.1186/s12916-020-01619-5

Anwar S, Nasrullah M, Hosen MJ (2020) COVID-19 and Bangladesh: challenges and how to address them. Front Public Health 8:154. https://doi.org/10.3389/fpubh.2020.00154

BBC (2020) BBC News Services, 22 June 2020. Homepage <https ://www.bbc.com/news/world-asia-53135626>. Accessed 24 Jun 2020

BBS (2015a) Economic census, 2013. Bangladesh Bureau of Statistic, Dhaka

BBS (2015b) Population and housing census, 2011, national report, vol 1. Bangladesh Bureau of Statistic, Dhaka
BBS (2015c) Population density and vulnerability - a challenge for sustainable development of Bangladesh, population monographvol 7. Bangladesh Bureau of Statistic, Dhaka

BBS (2015d) Populatiom distribution and internal migration in Bangladesh, Population Monograph: vol-6. Bangladesh Bureau of Statistic, Dhaka

BBS (2015e) International migrants from Bangladesh: socio-economic and regional characteristics, population Monograph: vol-3. Bangladesh Bureau of Statistic, Dhaka

BBS (2019) Final report on household income and expenditure survey 2016. Bangladesh Bureau of Statistic, Dhaka

Bergquist R, Rinaldi L (2020) COVID-19: pandemonium in our time. Geospat Health 15:880. https://doi.org/10.4081/gh.2020.880

Bodrud-Doza M, Shammi M, Bahlman L, Islam ARMT, Rahman MM (2020) Psychosocial and socio-economic crisis in Bangladesh due to COVID-19 pandemic: a perception-based assessment. Front Public Health 8:341. https://doi.org/10.3389/fpubh.2020.00341

Böhmer MM, Buchholz U, Corman VM, Hoch M, Katz K, Marosevic DV, Böhm S, Woudenberg T et al (2020) Investigation of a COVID-19 outbreak in Germany resulting from a single travelassociated primary case: a case series. Lancet Infect Dis. https:// doi.org/10.1016/S1473-3099(20)30314-5

Brody H (2003) Cholera, chloroform, and the science of medicine: a life of John Snow. Oxford University Press, Oxford, p 30 (ISBN 9780199747887)

CGTN (2020) A second wave of COVID-19 in China, China Global Television Network (CGTN), Beijing ICP prepared NO.160653103.https://news.cgtn.com/news/2020-06-19/A-second-wave-ofCOVID-19-in-China-RrSVGLFLeU/index.html. Acquired 24 Jun 2020

Chan YWD, Flasche S, Lam TLT et al (2020) Transmission dynamics, serial interval and epidemiology of COVID-19 diseases in Hong Kong under different control measures. Welcome Open Res 5:91. https://doi.org/10.12688/wellcomeopenres.15896.1

Chinazzi M, Davis JT, Ajelli M, Gioannini C, Litvinova M, Merler S, Pastore Y, Piontti A, Mu K, Rossi L, Sun K, Viboud C, Xiong X, Yu H, Halloran ME, Longini IM, Vespignani A (2020) The effect of travel restrictions on the spread of the 2019 novel coronavirus (COVID-19) outbreak. Science. https://doi.org/10.1126/ science.aba9757

Chowdhury R, Heng K, Shawon MSR, Goh G, Okonofua D, OchoaRosales C, Gonzalez-Jaramillo V, Bhuiya A, Reidpath D, Prathapan S, Shahzad S, Althaus CL, Gonzalez-Jaramillo N, Franco OH (2020) Dynamic interventions to control COVID-19 pandemic: a multivariate prediction modelling study comparing 16 worldwide countries. Eur J Epidemiol 35:389-399. https://doi. org/10.1007/s10654-020-00649-w

Cliff AD, Ord JK (1981) Spatial processes: models and applications. Pion, New York

DGHS (2020) Daily Press release, 2020, Directorate General of Health Services (DGHS), Ministry of Health and family welfare, Bangladesh. https://corona.gov.bd/press-release

Fan J, Liu X, Pan W, Douglas MW, Bao S (2020) Epidemiology of coronavirus disease in Gansu Province, China. Emerg Infect Dis 26(6)

Ferguson NM, Laydon D, Nedjati-Gilani G et al (2020) Impact of nonpharmaceutical interventions (NPIs) to reduce COVID19 mortality and healthcare demand. London: WHO Collaborating Centre for Infectious Disease Modelling MRC Centre for Global Infectious Disease Analysis Abdul Latif Jameel Institute for Disease and Emergency Analytics Imperial College London

Gatto M, Bertuzzob E, Maria L, Miccolid S, Carraroe L, Casagrandia R, Rinaldog A (2020) Spread and dynamics of the COVID-19 epidemic in Italy: effects of emergency containment measures. PNAS. https://doi.org/10.1073/pnas.2004978117/-/DCSuppleme ntal.y 
Getis A, Ord JK (1992) The analysis of spatial association by use of distance statistics. Geogr Anal 24(3):189-206

Ghosh K, Sengupta N, Manna D, De SK (2020) Inter-state transmission potential and vulnerability of COVID-19 in India. Progress Disaster Sci 7:100114

Hossain A, Rana J, Benzadid S, Ahsan GU (2020) COVID-19 and Bangladesh-weekly report, North South University, Dhaka, Bangladesh. doi: 10.13140/RG.2.2.34011.52000

Huang R, Liu M, Ding Y (2020) Spatial-temporal distribution of COVID-19 in China and its prediction: a data-driven modeling analysis. J Infect Dev Ctries 14(3):246-253. https://doi. org/10.3855/jidc. 12585

Hui D et al (2020) The continuing 2019-nCoV epidemic threat of novel coronavirus to global health-the latest 2019 novel coronavirus outbreak in Wuhan, China. Int J Infect Dis 91:264-266

IEDCR (2020) COVID-19 Status for Bangladesh, Institute of Epidemiology, Disease Control and Research (IEDCR). https://www. iedcr.gov.bd/

IndexMundi (2020) IndexMundi data portal, https://www.indexmundi .com/coronavirus/country/fr

Jahan Y, Rahman S, Rahman A (2020) COVID-19: a case report from Bangladesh perspective. Respir Med Case Rep 30:101068

Jia JS, Lu X, Yuan Y, Xu G, Jia J, Christakis NA (2020) Population flow drives spatio-temporal distribution of COVID-19 in China. Nature. https://doi.org/10.1038/s41586-020-2284-y

Kamel-Boulos MN, Geraghty EM (2020) Geographical tracking and mapping of coronavirus disease COVID-19/severe acute respiratory syndrome coronavirus 2 (SARS-CoV-2) epidemic and associated events around the world: how 21st century GIS technologies are supporting the global fight against outbreaks and epidemics. Int J Health Geogr 19:8. https://doi.org/10.1186/s12942-02000202-8

Kandwal R, Garg PK, Garg RD (2009) Health GIS and HIV/ AIDS studies: Perspective and retrospective. J Biomed Inform 42(4):748-755. https://doi.org/10.1016/j.jbi.2009.04.008

Kang D, Choi H, Kim J-H, Choi J (2020) Spatial epidemic dynamics of the COVID-19 outbreak in China. Int J Infect Dis 94:96-102. https://doi.org/10.1016/j.ijid.2020.03.076

Kenner R (2010) Influenza 1918. American Experience. Season 10. Episode 5. PBS. WGBH. Transcript

Khan MHA, Gupta SD, Hasan T, Chowdhury DR, Hasan MM (2020a) COVID-19: A threat to human existence. IOSR J Human Soc Sci 25(5):53-57. https://doi.org/10.9790/0837-25050953557

Khan MHR, Howlader T, Islam MM (2020b) Battling the COVID19 pandemic: is Bangladesh prepared? medRxiv. https://doi. org/10.1101/2020.04.29.20084236

Kirby RS, Delmelle E, Eberth JM (2017) Advances in spatial epidemiology and geographic information systems. Ann Epidemiol 27(1):1-9

Li X, Zhao X, Sun Y (2020) The lockdown of Hubei Province causing different transmission dynamics of the novel coronavirus (2019-nCoV) in Wuhan and Beijing. medRxiv. https://doi. org/10.1101/2020.02.09.20021477

Lyseen AK, Nohr C, Sorensen EM, Gudes O, Geraghty EM, Shaw NT, Bivona-Tellez C (2014) A review and framework for categorizing current research and development in health related Geographical Information Systems (GIS) studies. Yearb Med Inform 9:110-124. https://doi.org/10.15265/IY-2014-0008

Malczewski J (2006) GIS-based multi criteria decision analysis: a survey of the literature. Int J Geogr Inform Sci 20(7):249-268

Masrur A, Yu M, Luo W, Dewan A (2020) Space-time patterns, change, and propagation of COVID-19 risk relative to the intervention scenarios in Bangladesh. Int J Environ Res Public Health 17:5911. https://doi.org/10.3390/ijerph17165911

Melin P, Monica JC, Sanchez D, Castillo O (2020) Analysis of spatial spread relationships of coronavirus (COVID-19) pandemic in the world using self organizing maps. Chaos Solitons Fractals. https ://doi.org/10.1016/j.chaos.2020.109917

Meyer S, Held L, Hohle M (2017) Spatio-temporal analysis of epidemic phenomena using the $r$ package surveillance. J Stat Softw 77(11): $1-55$

Mishra SV, Gayen A, Haque SKM (2020) COVID-19 and urban vulnerability in India. Habitat Int 103:102230

Mo C, Tan D, Mai T, Bei C, Qin J, Pang W, Zhang Z (2020) An analysis of spatiotemporal pattern for COIVD-19 in China based on space-time cube. J Med Virol 92:1587-1595

Murugesan B, Karuppannan S, Mengistie AT, Ranganathan M, Gopalakrishnan G (2020) Distribution and trend analysis of COVID-19 in India: geospatial approach. J Geogr Stud 4(1):1-9

Penerliev M, Petkov V (2020) Geodemographic aspects of COVID-19. Espaço Econ. https://doi.org/10.4000/espacoeconomia

Rahman MR, Lateh H (2016a) Spatio-temporal analysis of warming in Bangladesh using recent observed temperature data and GIS. Clim Dyn 46:2943-2960. https://doi.org/10.1007/s00382-015-2742-7

Rahman MR, Lateh H (2016b) Meteorological drought in bangladesh: assessing analysing and hazard mapping using SPI GIS and monthly rainfall data. Environ Earth Sci. https://doi.org/10.1007/ s12665-016-5829-5

Rahman MR, Lateh H (2017) Climate change in Bangladesh: a spatio-temporal analysis and simulation of recent temperature and rainfall data using GIS and time series analysis model. Theoret Appl Climatol 128(1-2):27-41. https://doi.org/10.1007/s0070 4-015-1688-3

Rahman MR, Saha SK (2007) Flood hazard zonation-a GIS aided multicriteria evaluation approach (MCE) with remotely sensed data. Int J Geoinform 3(3):25-37

Rahman MR, Saha SK (2008) Remote sensing, spatial multi criteria evaluation (SMCE) and analytical hierarchy process (AHP) in optimal cropping pattern planning for a flood prone area. J Spat Sci 53(2):161-177

Rahman MR, Shi ZH, Chongfa C (2009) Soil erosion hazard evaluation-an integrated use of remote sensing, GIS and statistical approaches with biophysical parameters towards management strategies. Ecol Model 220(13-14):1724-1734

Rahman MR, Shi ZH, Chongfa C (2014) Assessing regional environmental quality by integrated use of remote sensing, GIS, and spatial multi-criteria evaluation for prioritization of environmental restoration. Environ Monit Assess 186(11):6993-7009. https:// doi.org/10.1007/s10661-014-3905-4

Rahman MR, Shi ZH, Chongfa C, Dun Z (2015) Assessing soil erosion hazard-a raster based GIS approach with spatial principal component analysis (SPCA). Earth Sci Inf 8:853-865. https://doi. org/10.1007/s12145-015-0219-1

Ramírez-Aldana R, Gomez-Verjan JC, BelloChavolla OY (2020) Spatial analysis of COVID-19 spread in Iran: insights into geographical and 2 structural transmission determinants at a province level. medRxiv. https://doi.org/10.1101/2020.04.19.20071605.t

Roy S, Bhunia GS, Shit PK (2020) Spatial prediction of COVID-19 epidemic using ARIMA techniques in India. Model Earth Syst Environ. https://doi.org/10.1007/s40808-020-00890-y

Saaty TL (1977) A scaling method for priorities in hierarchical structures. J Math Psychol 15:234-281

Sakamoto M, Begum S, Ahmed T (2020) Vulnerabilities to COVID-19 in Bangladesh and a reconsideration of sustainable development goals. Sustainability 12:5296. https://doi.org/10.3390/su12135296

Statista (2020) Statista, Inc. 55 Broad Street; 30th floor New York, NY 10004, United States. Webpage https://www.statista.com/stati stics/1104645/covid19-testing-rate-select-countries-worldwide/

Tobler W (2020) A computer movie simulating urban growth in the detroit region. Econ Geogr 46(Supplement):234-240

UNDP (2015) What are the sustainable development goals. United Nations Development Programme (UNDP), https://www.undp. 
org/content/undp/en/home/sustainable-developmentgoals.html/. Accessed 26 Jan 2020

UNDP (2020) 2020 human development perspectives, COVID-19 and human development: assessing the crisis, envisioning the recovery, United Nations Development Programme (UNDP)

Voinsky I, Baristaite G, Gurwitz D (2020) Effects of age and sex on recovery from COVID-19: analysis of 5769 Israeli patients. J Infect. https://doi.org/10.1016/j.jinf.2020.05.026

Walker PG, Whittaker C, Watson O et al (2020) The global impact of COVID-19 and strategies for mitigation and suppression. London: WHO Collaborating Centre for Infectious Disease Modelling, MRC Centre for Global Infectious Disease Analysis, Abdul Latif Jameel Institute for Disease and Emergency Analytics, Imperial College London

WHO (2020a) Novel coronavirus-China, World Health Organization. Homepage https://www.who.int/csr/don/12-january-2020-novel -coronavirus-china

WHO (2020b) Naming the coronavirus disease (COVID-19) and the virus that causes it, technical guidance. World Health Organization, Geneva

WHO (2020c) Statement on the second meeting of the International Health Regulations (2005) Emergency Committee regarding the outbreak of novel coronavirus (2019-nCov). World Health Organization, Geneva

WHO (2020d) WHO Regional Office for Europe, World Health Organization, UN City, Marmorvej 51, DK-2100 Copenhagen Ø, Denmark, Homepage https://www.euro.who.int/en/health-topics/healt h-emergencies/coronaviruscovid19\#text=WHO\%2520announ ced\%2520COVID\%2D19,on\%252011\%2520March\%25202020>. Accessed 23 Jun 2020

WHO (2020e) WHO Coronavirus Disease (COVID-19) Dashboard, World Health Organization (COVID-19). Homepage https://covid 19.who.int/. Data acquired 6 Aug 2020

WHO (2020f) COVID-2019 Bangladesh situation reports. World Health Organization, Bangladesh, https://www.who.int/bangl adesh/emergencies/coronavirus-disease-(COVID-19)-update/ coronavirus-disease-(covid-2019)-bangladesh-situation-reports
Worldometer (2020) COVID-19 Coronavirus Pandemic. https://www. worldometers.info/coronavirus/

Wu Z, McGoogan JM (2020) Characteristics of and important lessons from the coronavirus disease 2019 (COVID-19) outbreak in China Summary of a report of 72314 cases from the Chinese Center for Disease Control and Prevention. JAMA. https://doi.org/10.1001/ jama.2020.2648

Xiong H, Yan H (2020) Simulating the infected population and spread trend of 2019-nCov under different policy by EIR model. medRxiv. https://doi.org/10.1101/2020.02.10.20021519

Xu X W, Wu X X, Jiang X G, Xu K J, Ying L J, Ma C L, Sheng J F et al (2020) Clinical findings in a group of patients infected with the 2019 novel coronavirus (SARS-Cov-2) outside of Wuhan, China: retrospective case series. BMJ 368

Zabir AA, Mahmud A, Islam MA, Antor SC, Yasmin F, Dasgupta A (2020) COVID-19 and food supply in Bangladesh: a review. SSRN. https://doi.org/10.2139/ssrn.3595967

Zhou C, Su F, Pei T, Zhang A, Dua Y, Luo B, Cao Z, Wang J, Yuan W, Zhu Y, Songa C, Chen J, Xu J, Li F, Ma T, Jiang L, Yan F, Yi J, Hu Y, Liao Y, Xiao H (2020) COVID-19: challenges to GIS with Big Data. Geogr Sustain 1:77-87

Zhu N, Zhang D, Wang W, Li X, Yang B et al (2020) A novel coronavirus from patients with pneumonia in China. N Engl J Med 382(8):727-733. https://doi.org/10.1056/NEJMoa2001017

Zhu X, Zhang A, Xu S, Jia P, Tan X, Tian J, Wei T, Quan Z, Yu J (2020b) Spatially explicit modeling of 2019-nCoV epidemic trend based on mobile phone data in mainland China. medRxiv. https:// doi.org/10.1101/2020.02.09.20021360

Publisher's Note Springer Nature remains neutral with regard to jurisdictional claims in published maps and institutional affiliations.

\section{Affiliations}

\section{Md. Rejaur Rahman ${ }^{1}$ (D) A. H. M. Hedayutul Islam ${ }^{1}$ - Md. Nazrul Islam²}

\author{
A. H. M. Hedayutul Islam \\ chandan_geography@yahoo.com \\ Md. Nazrul Islam \\ nazrul_geo@juniv.edu
}

1 Department of Geography and Environmental Studies, University of Rajshahi, Rajshahi 6205, Bangladesh

2 Department of Geography and Environment, Jahangirnagar University, Savar, Dhaka 1342, Bangladesh 